\title{
Analysis of Simulated and Experimental Noise Sources of Boeing 777 Main Gear Model via CLEAN in 3D
}

\author{
Patricio Ravetta ${ }^{1}$ \\ AVEC Inc., Blacksburg, Virginia 24060, USA \\ Mehdi R. Khorrami ${ }^{2}$ \\ NASA Langley Research Center, Hampton, Virginia, 23681, USA \\ and \\ Benedikt König, ${ }^{3}$ Ehab Fares ${ }^{4}$ \\ Exa GmbH, D-70563 Stuttgart, Germany
}

\begin{abstract}
Previously obtained experimental and simulated phased microphone array data for the NASA $26 \%$-scale model of a six-wheel main landing gear, with and without a toboggan-shaped noise reduction fairing, were analyzed using the CLEAN technique in three dimensions. The model is a high-fidelity representation of the Boeing 777-200 aircraft main landing gear. The reprocessing of the acoustic data was undertaken to address the deficiencies encountered in the source localization (beamform) maps and the integrated farfield spectra at mid to high frequencies in our previous study, where conventional array processing techniques were applied to the same data sets. Application of the CLEAN approach resulted in higher quality beamform maps with many of the previously observed side lobes either eliminated or suppressed significantly. As a result, integration of the experimentally and computationally based maps showed substantial improvement in the quality of the high-frequency spectral content relative to the corresponding spectra generated via conventional array processing, thus extending the good agreement achieved between the measured and predicted farfield spectra from $8 \mathrm{kHz}$ to $14 \mathrm{kHz}$ in model-scale frequencies. In addition, an examination of the simulated surface pressure fluctuations, off-surface flow field, and three-dimensionally constructed noise sources was conducted to determine the cause of the subtle discrepancies between measured and predicted sound pressure levels that occur in the $1,800 \mathrm{~Hz}$ to $4,000 \mathrm{~Hz}$ model-scale frequency range.
\end{abstract}

\section{Introduction}

Aircraft undercarriage, in particular the main landing gear on large civil transports, is a leading contributor to the total airframe-generated noise that affects communities during approach and landing [1]. These highly complex main landing gears possess a multitude of components with various sizes and shapes that reside in close proximity of each other; thus, identification of prominent sources of noise and the principal mechanisms that generate it remain elusive. Advanced, high-fidelity airframe noise simulation methodologies constitute a viable avenue for the system-level study of main gear noise sources and the development of effective noise mitigation technologies. However, the simulation of configurations with such extreme geometric complexity presents many challenges during resolution of the finer flow interactions among smaller gear elements responsible for the high-frequency component of the farfield noise spectrum.

\footnotetext{
${ }^{1}$ Co-owner, chief research engineer, senior member AIAA.

${ }^{2}$ Aerospace engineer, Computational AeroSciences Branch, associate fellow AIAA.

${ }^{3}$ Senior application engineer, Aerospace.

${ }^{4}$ Senior technical director, Aerospace, senior member AIAA.
}

American Institute of Aeronautics and Astronautics 
Recent studies that demonstrate the viability of numerical simulations in the prediction of system-level airframe noise for model- and full-scale complete Gulfstream aircraft [2-4] have paved the way for extending the same computational methodology to the main landing gear of a large civil transport. In our previous studies, the NASA 26\%-scale, main landing gear model of a Boeing 777-200 aircraft was used in isolation as a testbed [5,6]. The model is a replica of the main landing gear flown on the aircraft and has been tested in various wind tunnels, both in isolation [7-10] and installed configurations [11]. To generate the desired acoustic data for validating the simulations, the gear model, with and without a toboggan-shaped noise reduction fairing, was extensively tested again in the Virginia Tech Stability Wind Tunnel (VTSWT) in its anechoic configuration. The acoustic measurements were conducted using the facility's state-of-the-art 251-element microphone phased array designed and built by AVEC. Following the methodology outlined in Refs. [2-4], time-dependent computations of the $26 \%$-scale main gear model with and without the toboggan fairing installed were accomplished [6] using the Exa Corporation PowerFLOW ${ }^{\circledR}$ Lattice- $^{-}$ Boltzmann flow solver. An acoustic analogy approach based on permeable surfaces of the Ffowcs Williams and Hawkings (FWH) formulation was used to propagate the acoustic signals to the farfield and produce the synthetic pressure records at spatial locations corresponding to the phased array microphone positions in the VTSWT.

In the study of Ref. [6], both measured and simulated pressure records were processed in an identical manner via the conventional array beamforming technique. Integration of the respective maps produced farfield spectra that showed very good agreement between measurements and simulations up to the model-scale frequencies of $7-8 \mathrm{kHz}$. Above this mid-frequency range, the agreement deteriorated fast with both spectra displaying non-physical trends. Closer inspection of the measured and simulated beamform maps at frequencies beyond $8 \mathrm{kHz}$ revealed the appearance of many side lobes, which when integrated produced the observed abnormal sound pressure levels (SPL) even when the desired integration cutoff level was used. For the measured spectra, the side lobes and associated deterioration in the accuracy of the SPLs in the mid- to high-frequency range was partially attributed to degradation of the signal-tonoise ratio in the VTSWT. In the case of the synthetic spectra, this deterioration was attributed to insufficient spatial resolution during capture of the high-frequency content in the nearfield flow.

In this paper, we present additional aeroacoustic analyses of the measured and simulated data sets. To delineate some of the trends encountered at higher frequencies, both data sets have been reprocessed using the CLEAN array processing technique over a three-dimensional (3D) grid surrounding the entire landing gear model. The application of CLEAN has produced better quality beamform maps, resulting in the elimination or significant suppression of the side lobes encountered with conventional array processing technique. As a result, integration of the experimentally and computationally based beamform maps shows marked improvement in the SPLs at high frequencies and extends the good agreement achieved between measured and predicted farfield spectra from $8 \mathrm{kHz}$ to beyond $14 \mathrm{kHz}$ in modelscale frequencies.

\section{Main Landing Gear Model}

A full description of the 26\%-scale B777-200 main landing gear model (MLG) is provided in Ref. [5]. The highfidelity model features all the major gear components: strut, braces, torque link, cable harnesses, lock links, main door, wheels, and a host of other smaller subcomponents that include oleo lines, cables, wheel hubs, brake cylinders, and hydraulic valves. The images shown in Fig. 1 (reproduced from Ref. [5]) demonstrate the geometric complexity and detail of the gear model. The figure also provides the naming convention used throughout this paper. In Fig. 1, the landing gear is shown in its nominal orientation that corresponds to a truck angle of $13^{\circ}$ (toe-up) position relative to the incoming flow. Also taken from Ref. [5] are the images shown in Figs. 2 and 3 that display a bottom view of the gear in its baseline configuration and with toboggan fairing installed, respectively.

American Institute of Aeronautics and Astronautics 

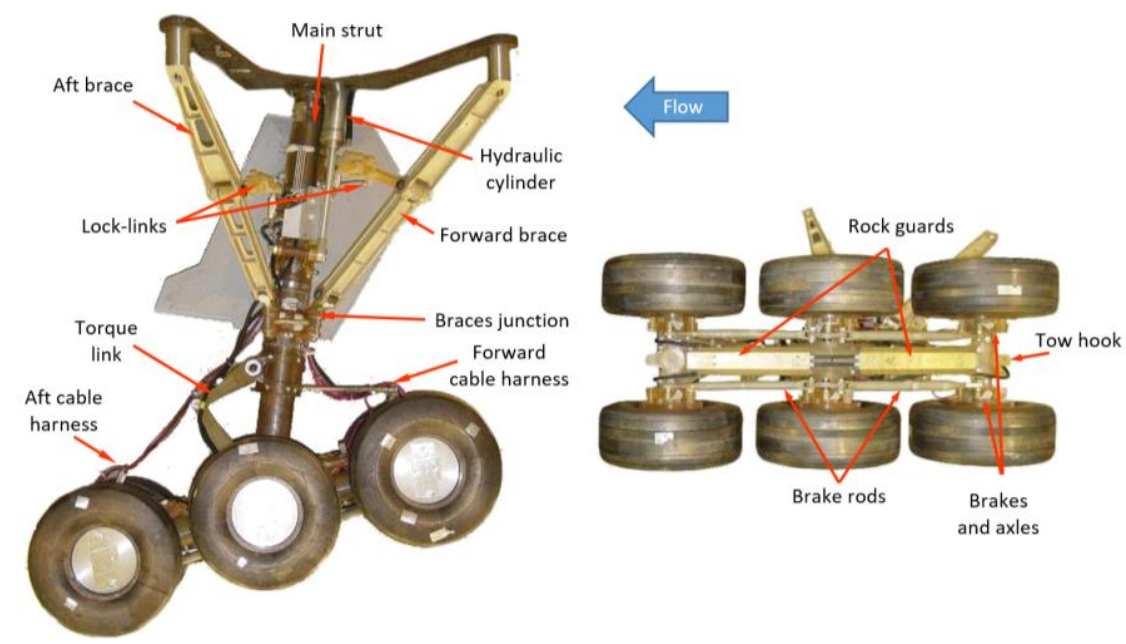

Fig. 1 Main landing gear images highlighting the names of major components (from Ref. [5]).

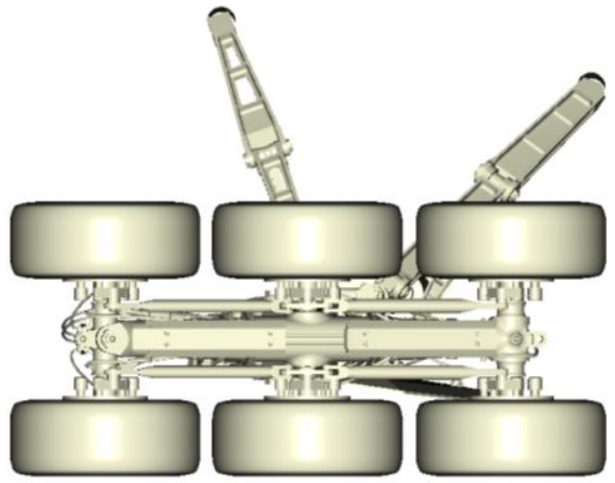

Fig. 2 Bottom view of the baseline MLG (from Ref. [5]).

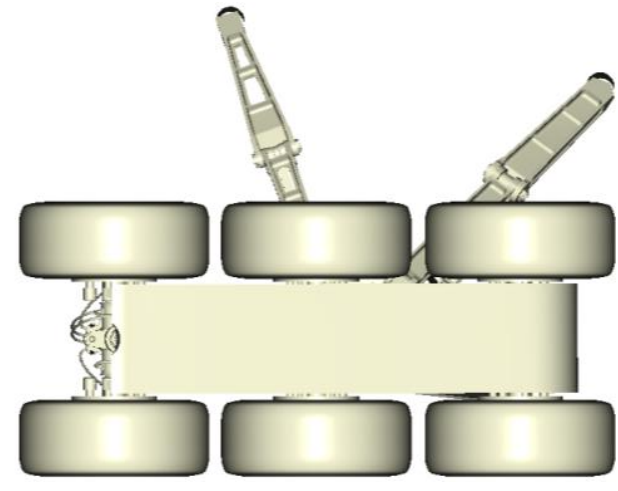

Fig. 3 Bottom view of the MLG with toboggan noise reduction concept (from Ref. [5]).

\section{Computational Approach}

The high-fidelity, unsteady, nearfield flow solutions were obtained using the high-subsonic version of the Exa PowerFLOW ${ }^{\circledR}$ lattice Boltzmann flow solver. To mimic the landing gear setup in the Virginia Tech tunnel in its anechoic configuration, the computations were performed for free air with the model mounted on a flat plate, duplicating the tunnel wall. All the computations were conducted at a Mach number (M) of 0.17, matching the highest speed used during the model tests. As part of a grid convergence study, the computations were performed using a series of grids that resolved a wheel diameter of the MLG of $\mathrm{D}=0.34 \mathrm{~m}$ with about 280,420 , and 630 voxels for the coarse, medium, and fine grids, respectively. Table 1, reproduced from Ref. [6], summarizes the size and attributes of the three grids used for the simulations. In the table, the computational cost is normalized by the number of CPU hours needed to compute 1 second of physical time in the flow.

Table 1. Grid size and computational cost for the simulation of 1 sec of physical flow time (from Ref. 6).

\begin{tabular}{lcccc} 
Resolution level & Voxels per $\boldsymbol{D}$ & Number of Voxels & Number of Surfels & kCPUh / $\mathbf{~ s e c}$ \\
\hline Coarse & 280 & $425 \times 10^{6}$ & $16 \times 10^{6}$ & 40 \\
Medium & 420 & $1360 \times 10^{6}$ & $27 \times 10^{6}$ & 210 \\
Fine & 630 & $4490 \times 10^{6}$ & $49 \times 10^{6}$ & 980
\end{tabular}

American Institute of Aeronautics and Astronautics 
An acoustic analogy approach based on the Ffowcs Williams and Hawkings (FWH) formulation [12] was used to propagate the computed nearfield fluctuations to the locations of the array's microphones in the far field. Following the recommendations of Ref. [6], only integration results based on a permeable surface surrounding the MLG and the mounting-plate, obtained from the fine-resolution simulations, were used in the present study. A full account of the simulation approach, including descriptions of the lattice-Boltzmann model, turbulence modelling, boundary conditions, meshing, convergence criteria, data sampling rate, and FWH integration used to generate the computational database, is given in Ref. [6].

\section{Data Analysis}

The FWH propagation approach described in Ref. [12] was used to obtain the time histories of the pressure at the same relative microphone locations used during the wind tunnel test. However, the differences in microphone location due to refraction (shear layer) effects caused by the presence of the wind tunnel Kevlar ${ }^{\circledR}$ walls were ignored in the computations. To eliminate any potential discrepancies related to the processing algorithms used by AVEC and EXA, both measured and simulated pressure records were beamformed and post-processed using the CLEAN technique [13], which is available within AVEC's Phased Array software suite. Although the measured and simulated data had different sampling frequencies, the spectral resolution for both sets was matched to allow a direct comparison of the narrowband acoustic maps and their corresponding integrated results.

Since the shear layer and $\mathrm{Kevlar}^{\circledR}$ walls introduce transmission losses, the experimental acoustic data had to be corrected to account for these effects. The corrections used are based on measurements performed in the empty wind tunnel described in Ref. [14].

The beamforming and deconvolution processes were performed over a 3D grid surrounding the landing gear with a resolution of $1 \mathrm{~cm}$ in the plane parallel to the array, and $5 \mathrm{~cm}$ in the direction normal to it. Diagonal removal beamforming was used to reduce the impact of uncorrelated noise. The array-integrated spectra were computed for the entire 3D grid surrounding the landing gear. For conventional beamforming results, the procedure in Ref. [15] was followed, that is, normalization by the point spread function (PSF) for a source at the center of the 3D grid, accounting for diagonal removal, and application of a cutoff level to reduce the contribution from sidelobes. To obtain the integrated spectra from CLEAN results with a "zero-resolution", all the sources within the cutoff level were added. Since the acoustic maps generated via CLEAN show a better array signal-to-noise ratio (SNR), a cutoff larger than that used for integration of conventional beamforming results (10 dB instead of $5 \mathrm{~dB}$ ) was used. Since additional sources are accounted for with the higher cutoff, more accurate integration results are obtained. Moreover, the integration of CLEAN results is more accurate due to elimination of the inaccuracies introduced by integrating a large 3D grid with sources throughout its volume (i.e., because the PSF used for normalization is at the center of the integration region, in this case the entire grid surrounding the model). CLEAN results were also obtained with an artificial resolution of $5 \mathrm{~cm}$ (for all frequencies) for ease of visualization of the acoustic maps. The CLEAN beamform maps presented in the next section were obtained with this resolution.

\section{Results and Discussion}

The main objective of the acoustic maps presented in this section is to demonstrate the improvement in the array SNR (the difference in level between the loudest source and the worst sidelobe) when CLEAN is used. As mentioned in the previous section, increasing the SNR allows use of a larger cutoff, which results in more accurate integrated levels. Fig. 4a shows a typical experimental, overhead acoustic map for conventional beamforming (CB) results at 2 $\mathrm{kHz}$. The corresponding CLEAN results over the same plane are shown in Fig. 4b. Note that, for this plane, the CLEAN sources on the gear's center and forward axles show lower levels or disappear altogether. This is a consequence of the improved resolution in the vertical direction (normal to the array plane) when using the CLEAN technique. If the entire set of two-dimensional (2D) planes is plotted and the contours in dark blue are hidden, the maps presented in Fig. 4c and Fig. 4d are obtained for CB and CLEAN, respectively. These "3D" maps provide a more meaningful visualization of the sources in the entire 3D grid while improving the assessment of the potential impact of sidelobes on the integration results. The latter is the main goal of the maps shown throughout this section.

As presented in

Fig. 5 for a frequency of $5 \mathrm{kHz}$, the resolution of the CB results improves but sidelobes became more prominent. As a consequence, the maximum integration cutoff that can be used without introducing errors is reduced (a value of $5 \mathrm{~dB}$ was used in our previous studies). As frequency increases, the presence of sidelobes reaches a point for which the integration of $\mathrm{CB}$ results is no longer accurate even when using a very small cutoff level. A sample CB map for a

American Institute of Aeronautics and Astronautics 
frequency of $14 \mathrm{kHz}$ is shown in Fig. 6a. In contrast, the CLEAN results in Fig. 6b clearly show that no spurious sources are observed within $10 \mathrm{~dB}$ of the peak level. Therefore, using a cutoff of $10 \mathrm{~dB}$, one would expect the integration results to accurately quantify the experimental noise sources up to at least this frequency.

The acoustic maps presented so far where obtained in narrowband using a frequency resolution of $50 \mathrm{~Hz}$. If acoustic maps in $1 / 24^{\text {th }}$ octave bands are generated from the narrowband maps, the relative levels of spurious sources is further reduced (see Fig. 7), and therefore the frequency range for which integration (in $1 / 24^{\text {th }}$ octave bands) is accurate could be extended. However, narrowband analysis was mainly used because the overall objective of this work was to perform an exhaustive comparison of the experimental and simulated sources.

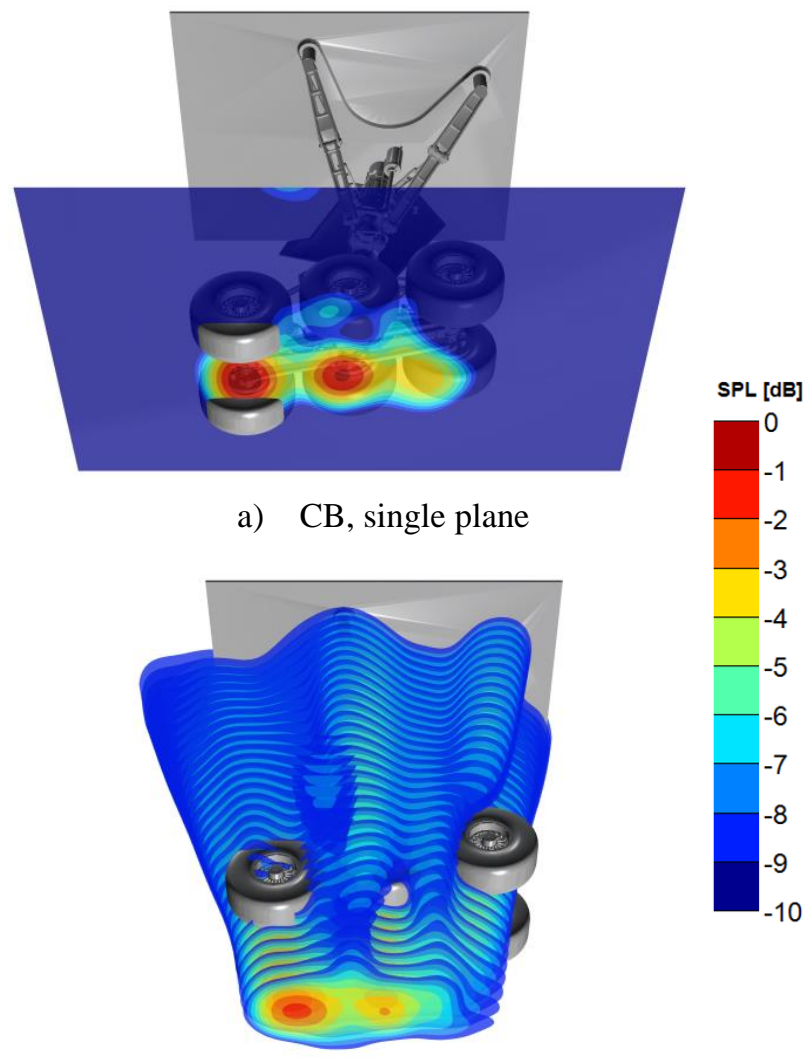

c) $\mathrm{CB}$

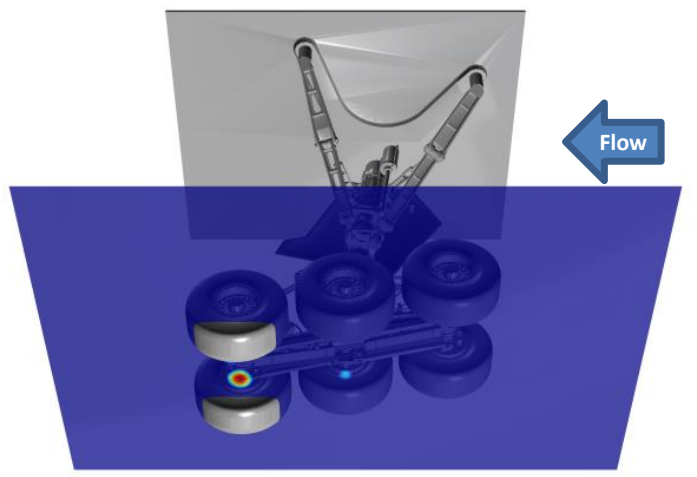

b) CLEAN, single plane

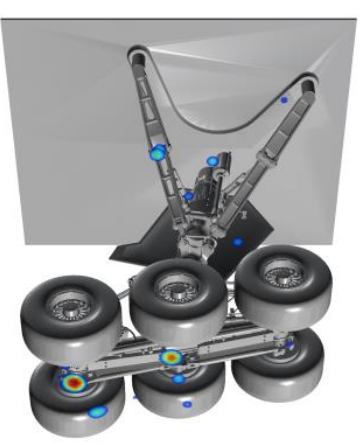

d) CLEAN

Fig. 4 Experimental narrowband beamform maps in overhead direction, $f=2 \mathrm{kHz}$.

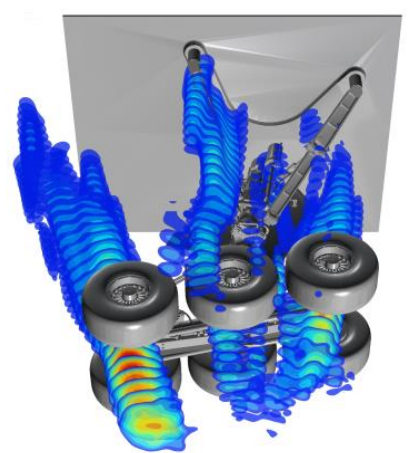

a) $\mathrm{CB}$

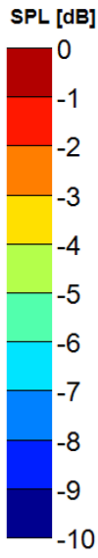

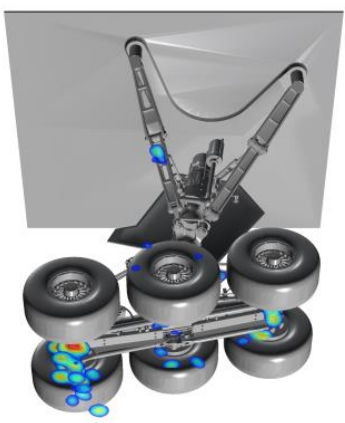

b) CLEAN 
Fig. 5 Experimental narrowband beamform maps in overhead direction, $f=5 \mathrm{kHz}$.

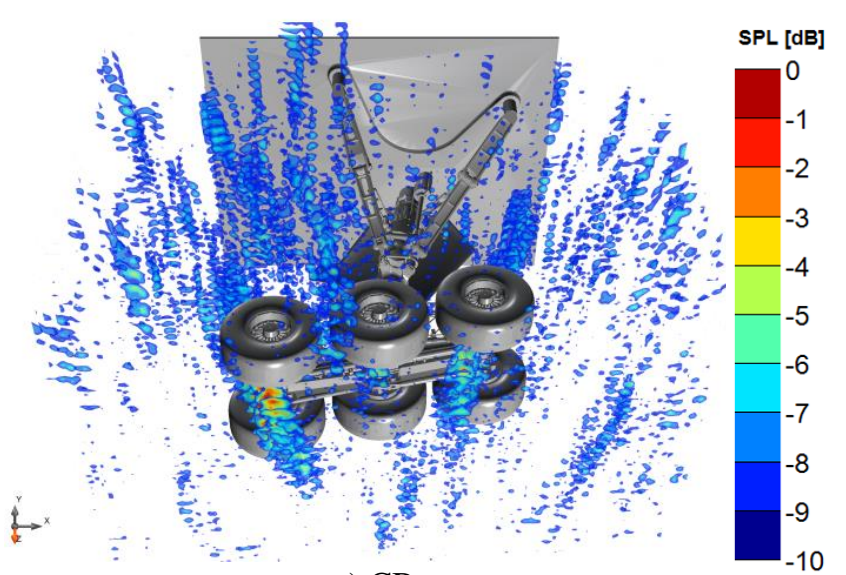

a) $\mathrm{CB}$

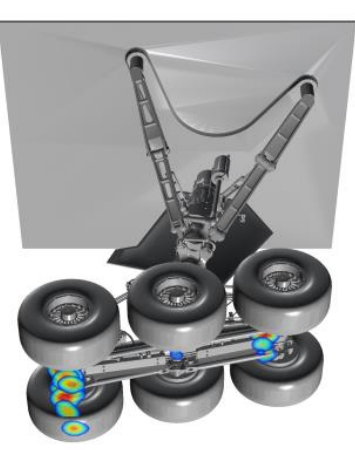

b) CLEAN

Fig. 6 Experimental narrowband beamform maps in overhead direction, $f=14 \mathrm{kHz}$.

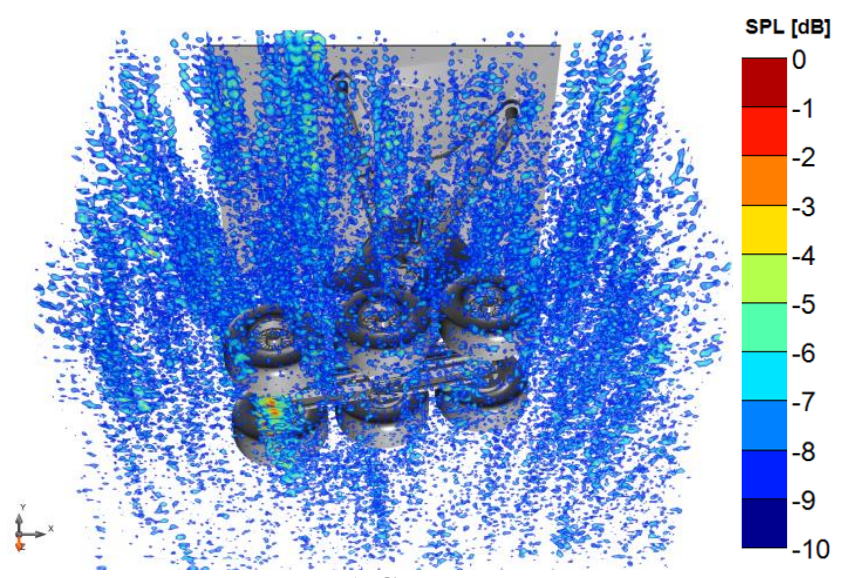

a) $\mathrm{CB}$
Fig. 7 Experimental $\left(1 / \mathbf{2 4}^{\text {th }}\right.$ octave bands) beamform maps in overhead direction, $19 \mathrm{kHz}$.

Comparisons of CB and CLEAN narrowband acoustic maps for the simulated data are presented in Fig. 8 through Fig. 10. As can be seen from these figures, CLEAN results for the simulated data provide a marked improvement over the CB results, particularly at higher frequencies. Since some spurious sources are already present, the maximum frequency for which the simulated CLEAN maps can be accurately integrated is slightly lower than the one for the experimental results. As shown earlier for the experimental results, the CLEAN maps in $1 / 24^{\text {th }}$ octave bands do not show as many spurious sources (see Fig. 11). Therefore, they can still be used for noise source identification and to obtain accurate octave band integrated spectra levels for higher frequencies.

The integrated spectra for the gear in its baseline configuration obtained from CB and CLEAN processing of the simulated and experimental data are shown in Fig. 12a. Since the contamination from spurious sources was significantly reduced for CLEAN results, the integration no longer shows the artificial increase in sound pressure levels (SPL) at high frequencies. The CLEAN results (solid lines) show a clear improvement at high frequencies when compared to CB results (dashed lines). The reduction in CLEAN levels relative to CB results at other frequencies is due to the location of the actual sources relative to the center of the scanning grid and to the manner in which the PSF is accounted for in CB integration. Since the PSF normalization is not used in CLEAN (the effect of the PSF is already accounted for during deconvolution), the integration is more accurate. The integrated spectra comparison for the configuration with the toboggan fairing installed is shown in Fig. 12b. Although there are still some subtle differences between experimental and simulated results, the measured and predicted integrated spectra using CLEAN are now in better agreement. This is particularly evident when the noise reduction attained with the toboggan fairing is compared 
instead, as shown in Fig. 13. Similar trends are observed when comparing the integrated spectra using CB and CLEAN for the sideline results, shown in Fig. 14. Note from Fig. 14a that a 2,600 Hz tone that appears in the simulated spectra is not present in the measurements. As mentioned in Ref. [6], this tone is associated with the forward cable harness. We must point out that there is a degree of uncertainty regarding the spatial position of the flexible cables that go through the harness. As a result, duplication of their position within the rigid representation in the CAD model is very difficult - even small deviations in the orientation of the cables relative to the incoming flow may give rise to different noise signatures.

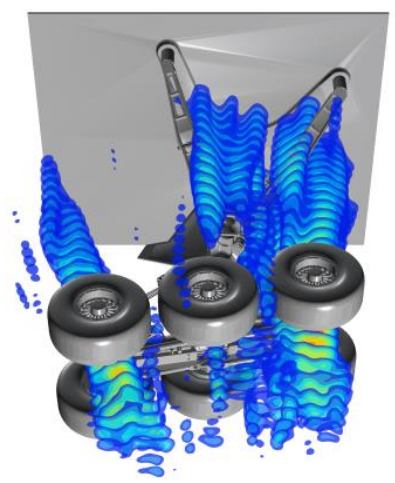

a) $\mathrm{CB}$

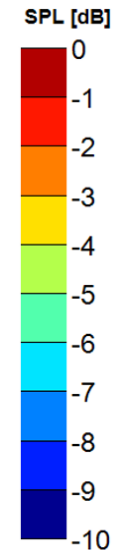

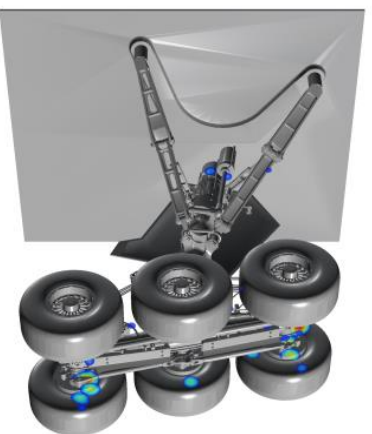

b) CLEAN

Fig. 8 Simulated narrowband beamform maps in overhead direction, $f=5 \mathrm{kHz}$.

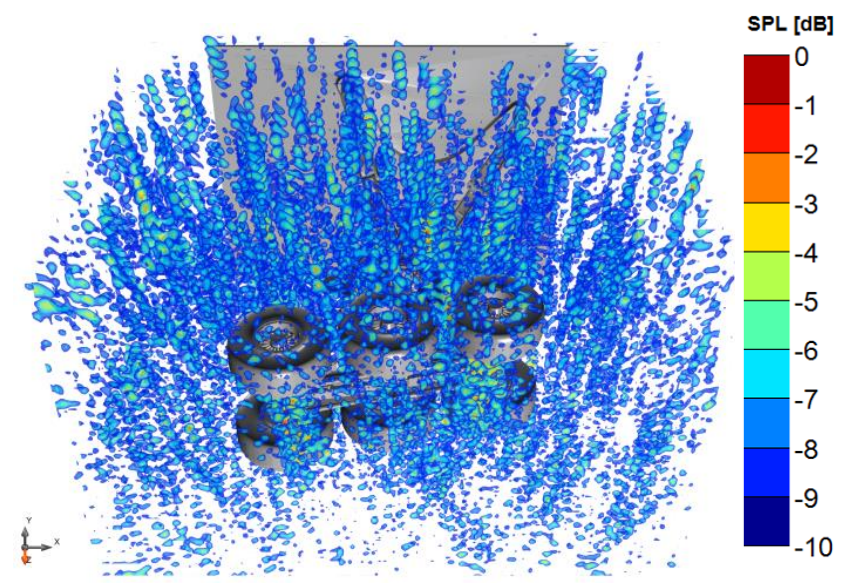

a) $\mathrm{CB}$
Fig. 9 Simulated narrowband beamform maps in overhead direction, $f=10 \mathrm{kHz}$.

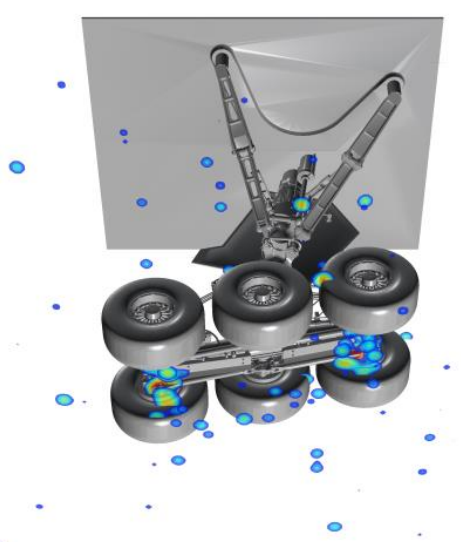

b) CLEAN

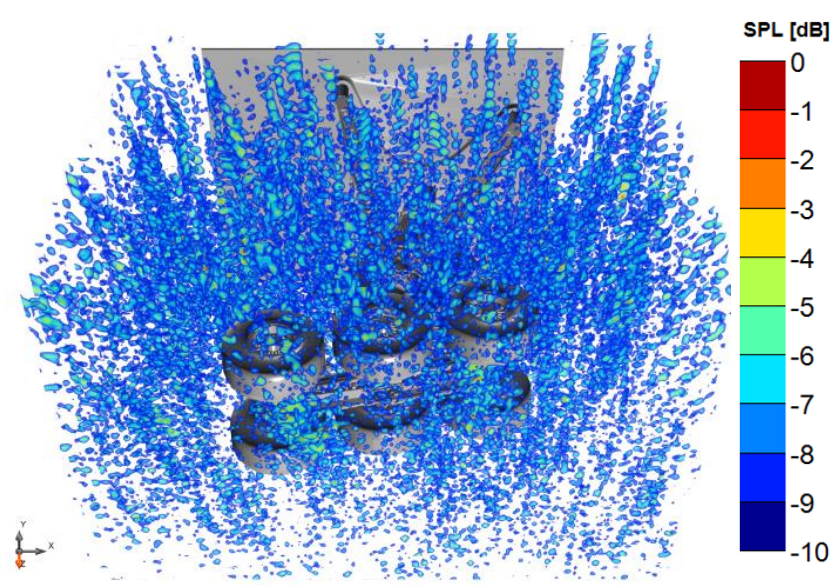

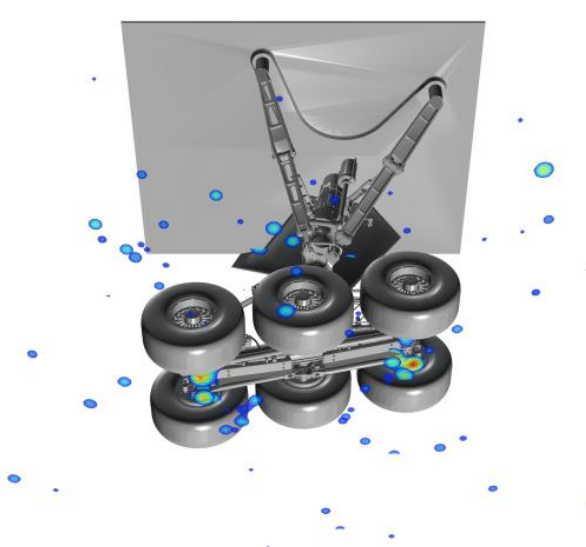

American Institute of Aeronautics and Astronautics 

a) $\mathrm{CB}$
b) CLEAN

Fig. 10 Simulated narrowband beamform maps in overhead direction, $f=12 \mathrm{kHz}$.

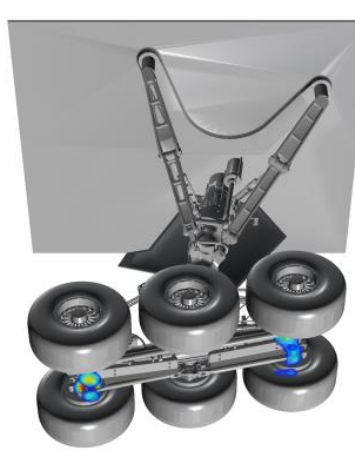

a) CLEAN $12.8 \mathrm{kHz}\left(1 / 24^{\text {th }}\right.$ octave band)

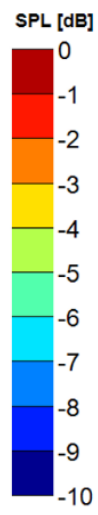

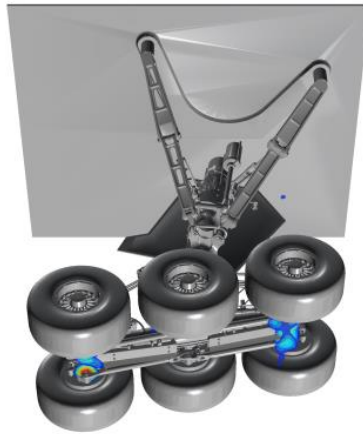

b) CLEAN $14 \mathrm{kHz}\left(1 / 12^{\text {th }}\right.$ octave band)

Fig. 11 Simulated beamform maps in overhead direction from CLEAN.

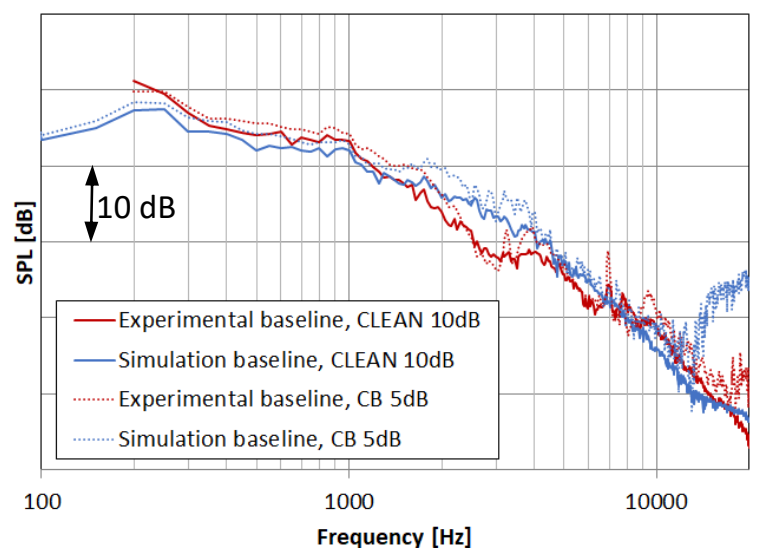

a) Baseline

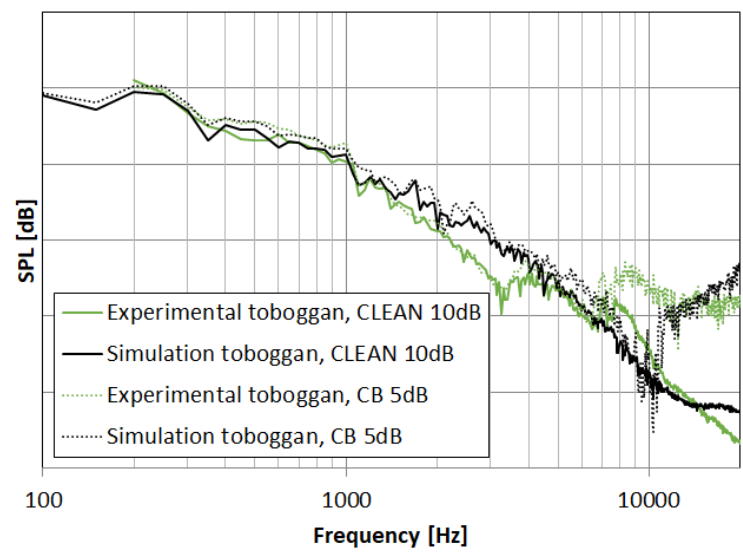

b) Toboggan fairing

Fig. 12 Farfield noise spectra in overhead direction obtained from integration of CLEAN and conventional beamforming contour maps.

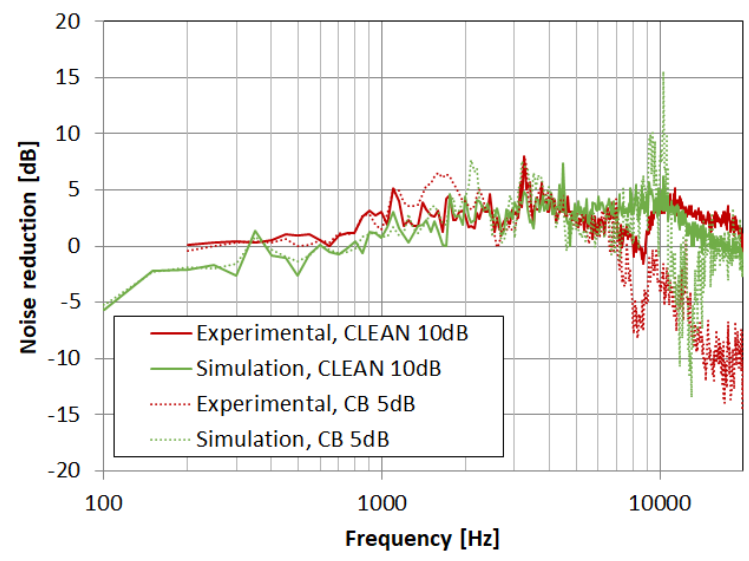

Fig. 13 Noise reduction achieved with toboggan fairing based on farfield noise spectra in overhead direction. Obtained from integration of CLEAN and conventional beamforming contour maps. 


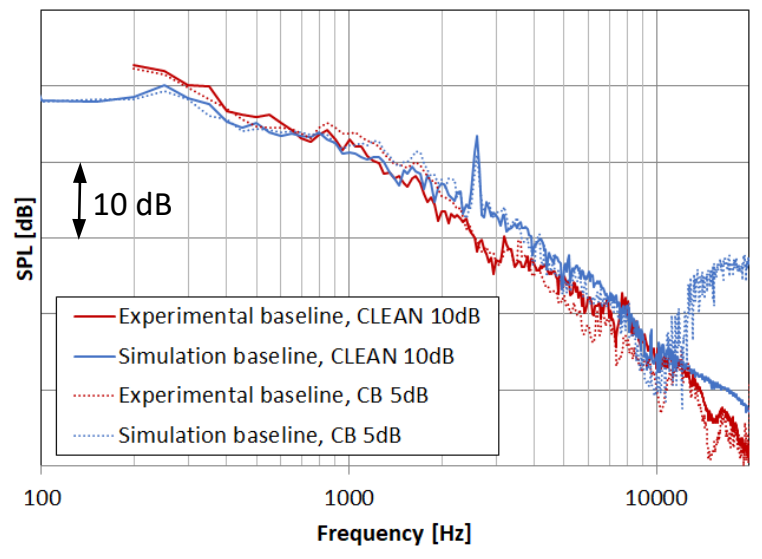

a) Baseline

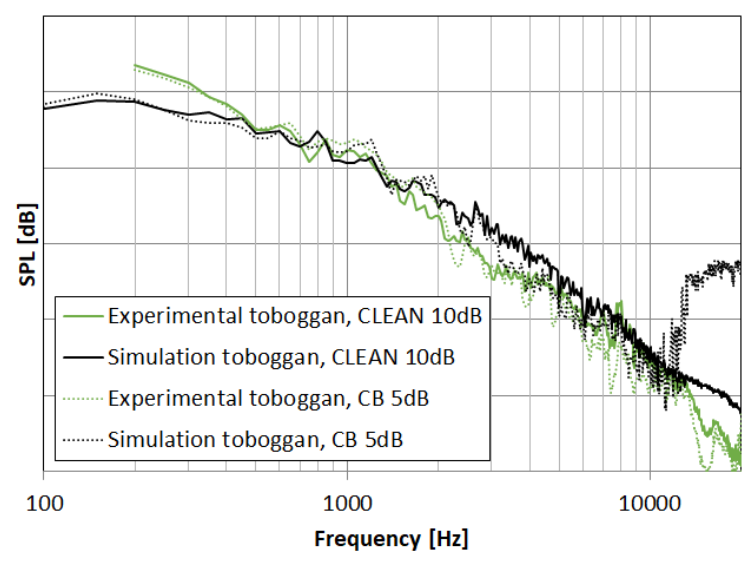

b) Toboggan

Fig. 14 Farfield noise spectra in sideline direction obtained from integration of CLEAN and conventional beamforming contour maps.

Given the extreme geometrical complexities associated with this gear model, the observed agreement between the two spectra over the entire frequency range is remarkable. The only notable differences occur at model-scale frequencies between $1.8 \mathrm{kHz}-4.0 \mathrm{kHz}$, where the measured spectrum displays a dip in SPL (faster roll-off) relative to the simulated spectrum followed by an increase in levels that regains the same spectral shape as the simulation.

Examination of the 3D source maps revealed that in the $1.8 \mathrm{kHz}-4 \mathrm{kHz}$ frequency range, nearly all dominant sources reside within the lower segment (truck) of the landing gear. In light of this finding and to highlight the differences between the experimental and simulated results, the source maps are presented for three planar grids that cut through the lower part of the gear model. Separation between planes in the $\mathrm{Z}$ (normal) direction is $0.1 \mathrm{~m}(10 \mathrm{~cm}$ ), as shown in Fig. 15. Note that the coordinate $\mathrm{Z}=0$ corresponds to the axis of the center axle.

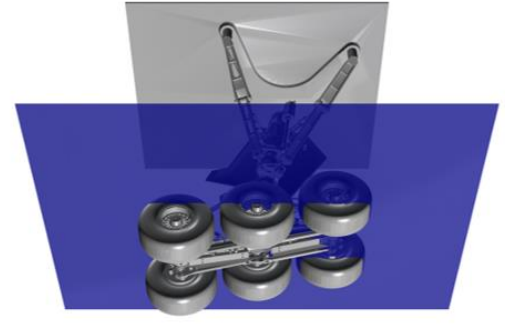

a) $Z=0 \mathrm{~m}$

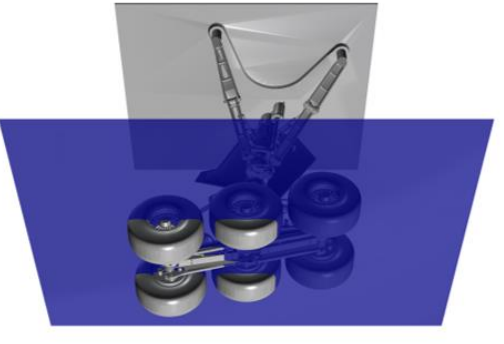

b) $\mathrm{Z}=0.1 \mathrm{~m}$

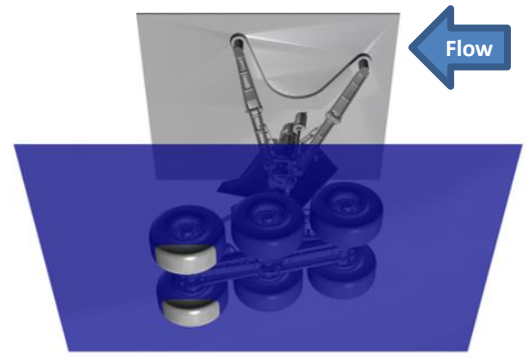

c) $\mathrm{Z}=0.2 \mathrm{~m}$

Fig. 15 Selected 2D planes used to highlight measured and simulated noise sources on landing gear truck.

Maps in $1 / 12^{\text {th }}$ octave band showing the sources at $1.4 \mathrm{kHz}$ for the baseline configuration are presented in Fig. 16. This frequency, which is outside the range mentioned above, was chosen to compare maps at a frequency where measured and simulated integrated spectra are in close agreement. In these maps and the maps that follow, the peak SPL in the entire 3D grid (whether measured or simulated) at each frequency is used to set the maximum level for both sets of contours, allowing a direct comparison of source strength. A $20 \mathrm{~dB}$ down cutoff range was used to highlight the majority of sources that could contribute to the integrated SPLs. Observe from Fig. 16 that the prominent source at $1.4 \mathrm{kHz}$ is associated with the aft axle (right column, $\mathrm{Z}=0.2 \mathrm{~m}$ plane) and that this source has similar strength for both measured and simulated maps. However, differences in the location of the sources start to emerge at $\mathrm{Z}=0.1$, where the gear aft axle remains the more dominant source, while the forward axle appears to be the stronger source in the simulated map. Beamform maps at $2.24 \mathrm{kHz}$ and $2.8 \mathrm{kHz}$ are presented in Figs. 17 and 18, respectively. These frequency bands represent values where the differences between measured and simulated integrated spectra are largest. Overall, the maps obtained from simulations provide a consistent picture regarding the location of the prominent sources on the truck - these sources are located mainly on the forward and aft axles. There are clear 
disparities in the source strength, however, with simulated sources showing 5-6 dB higher amplitude relative to those of the measured sources. This is exactly in line with the magnitude of differences in the spectral plots shown in Figure $12 \mathrm{~b}$, which were obtained from integration of the "zero-resolution" maps. The corresponding maps at $2.24 \mathrm{kHz}$ and $2.8 \mathrm{kHz}$ for the toboggan configuration are shown in Figs. 19 and 20, respectively. A stark difference between the magnitude of the measured and simulated sources can be observed from Fig. 19. The maps obtained from the measurements indicate the absence of any prominent source within $10-15 \mathrm{~dB}$ of the simulated sources. The fact that the measured and simulated integrated spectra (Fig. 12b) at this frequency are within $5 \mathrm{~dB}$ of each other suggests that the primary sources may reside on the upper part of the gear and not the truck. At $2.8 \mathrm{kHz}$ (Fig. 20), as in the baseline case, the primary sources present in the experimental maps are also captured by the simulation, albeit with a 6-7 dB over prediction of source strength. The simulated maps also show the presence of additional sources, the forward right wheel/brake assembly being the most prominent.

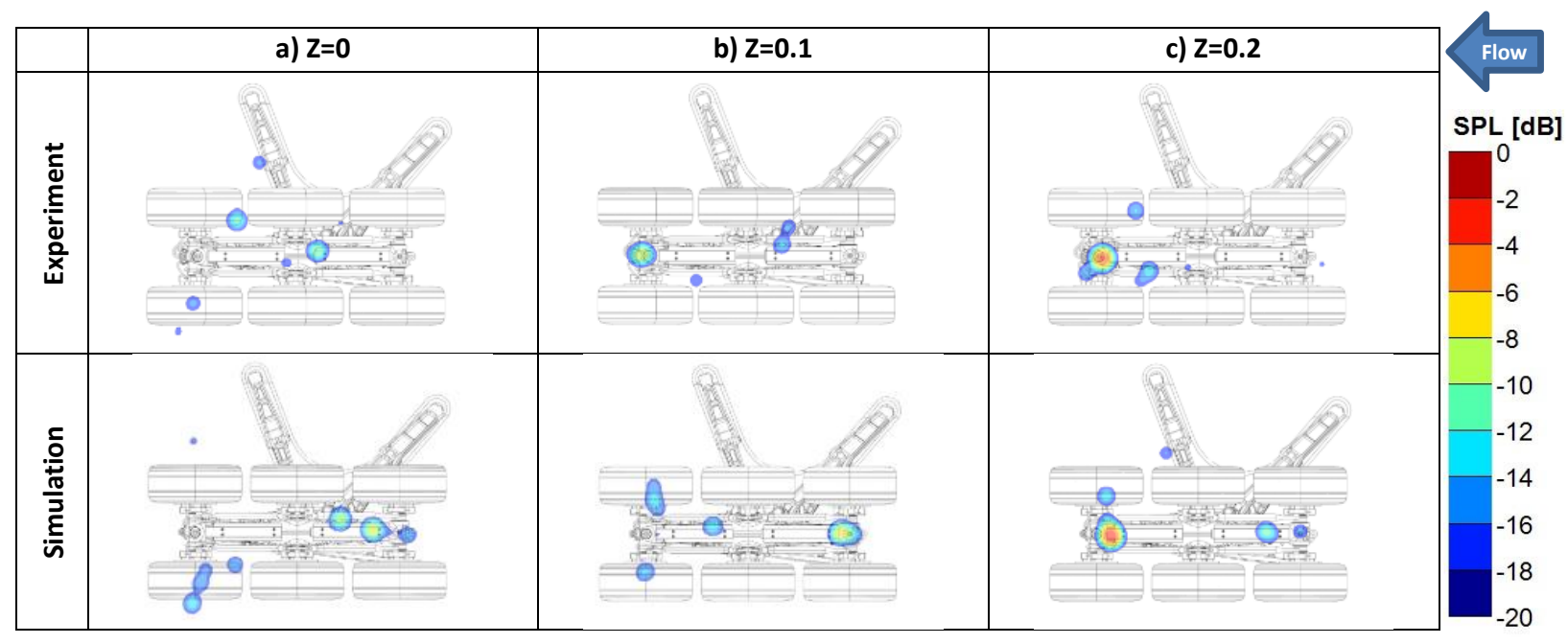

Fig. 16 Experimental and simulated (1/12th octave band) beamform maps for baseline configuration in overhead direction from CLEAN, $f=1.4 \mathrm{kHz}$.

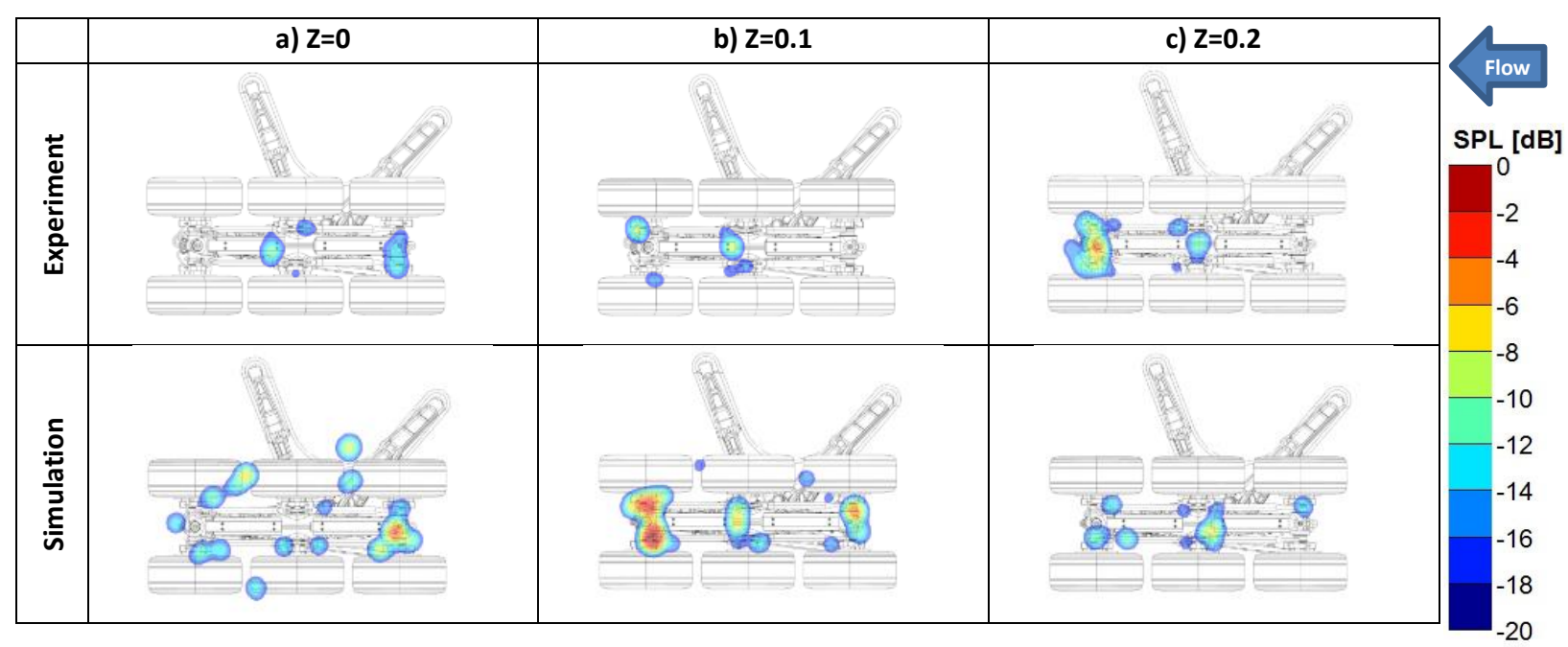

Fig. 17 Experimental and simulated (1/12th octave band) beamform maps for baseline configuration in overhead direction from CLEAN, $f=2.24 \mathrm{kHz}$. 


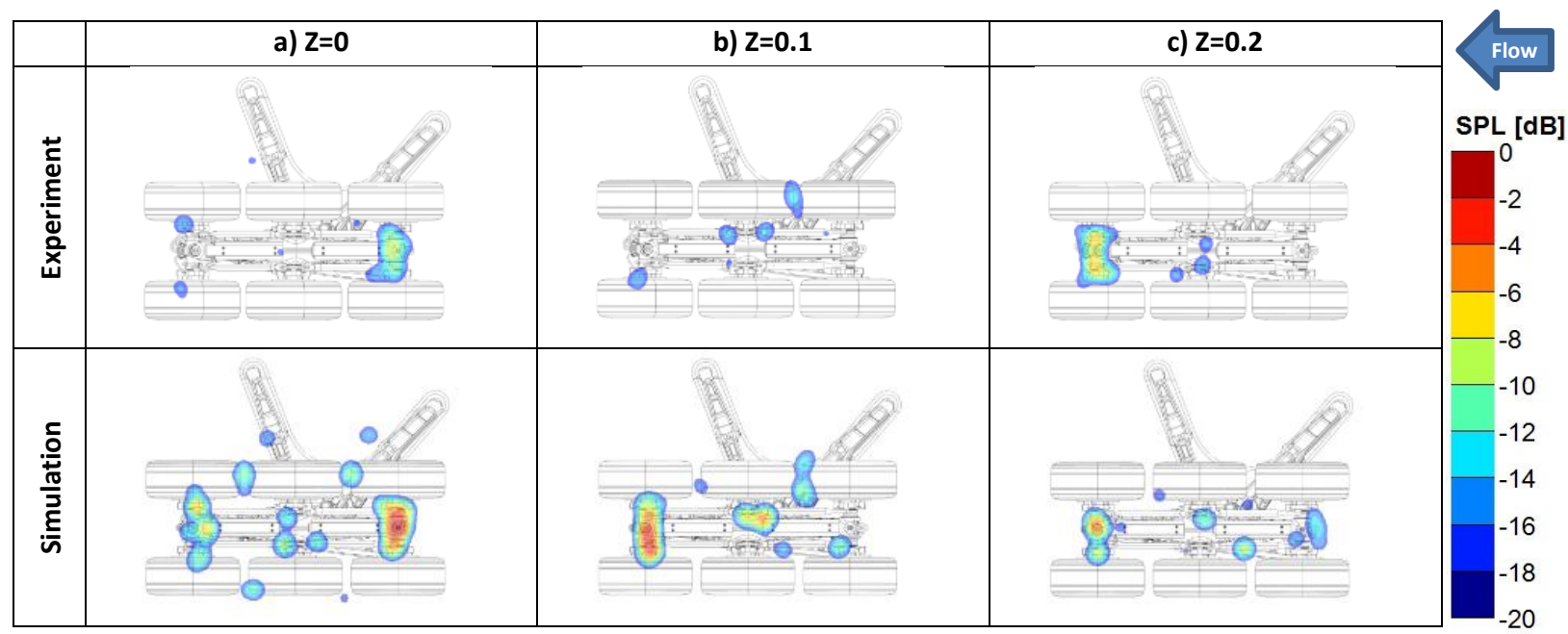

Fig. 18 Experimental and simulated (1/12th octave band) beamform maps for baseline configuration in overhead direction from CLEAN, $f=2.8 \mathrm{kHz}$.

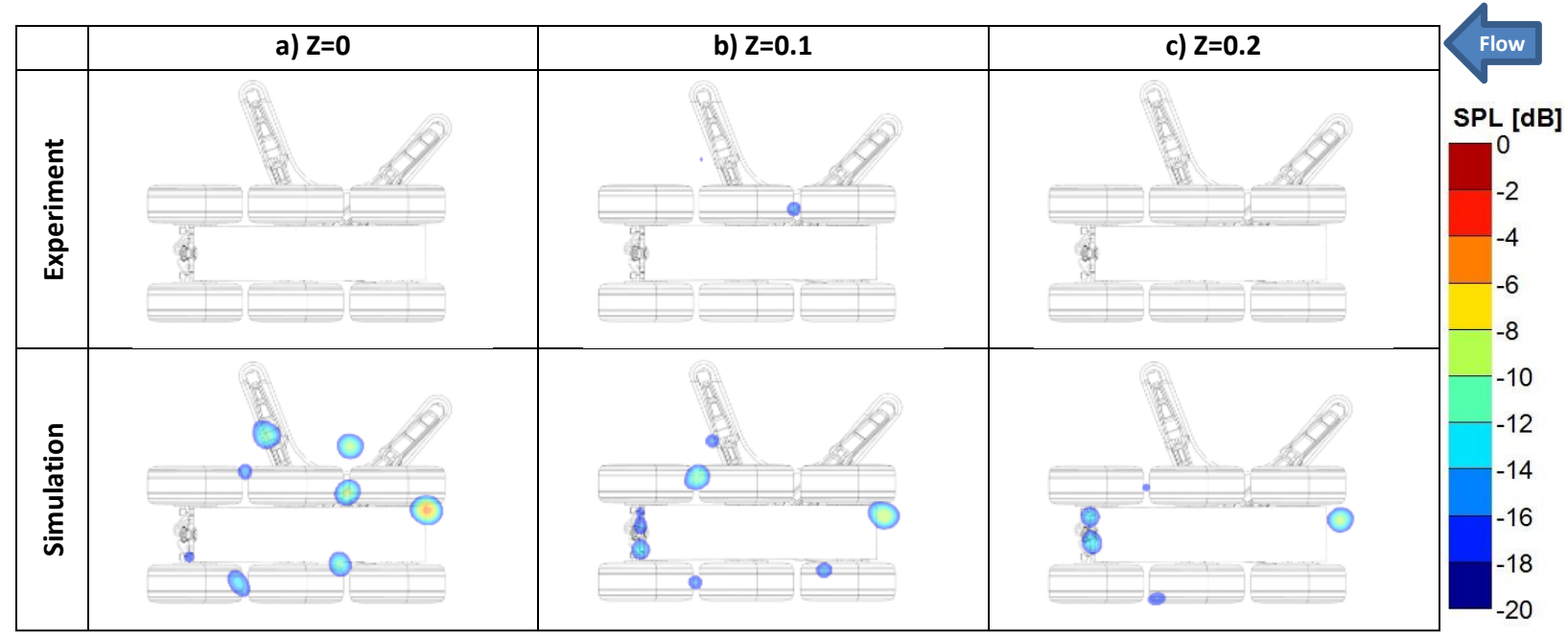

Fig. 19 Experimental and simulated (1/12th octave band) beamform maps with toboggan installed in overhead direction from CLEAN, $f=2.24 \mathrm{kHz}$. 


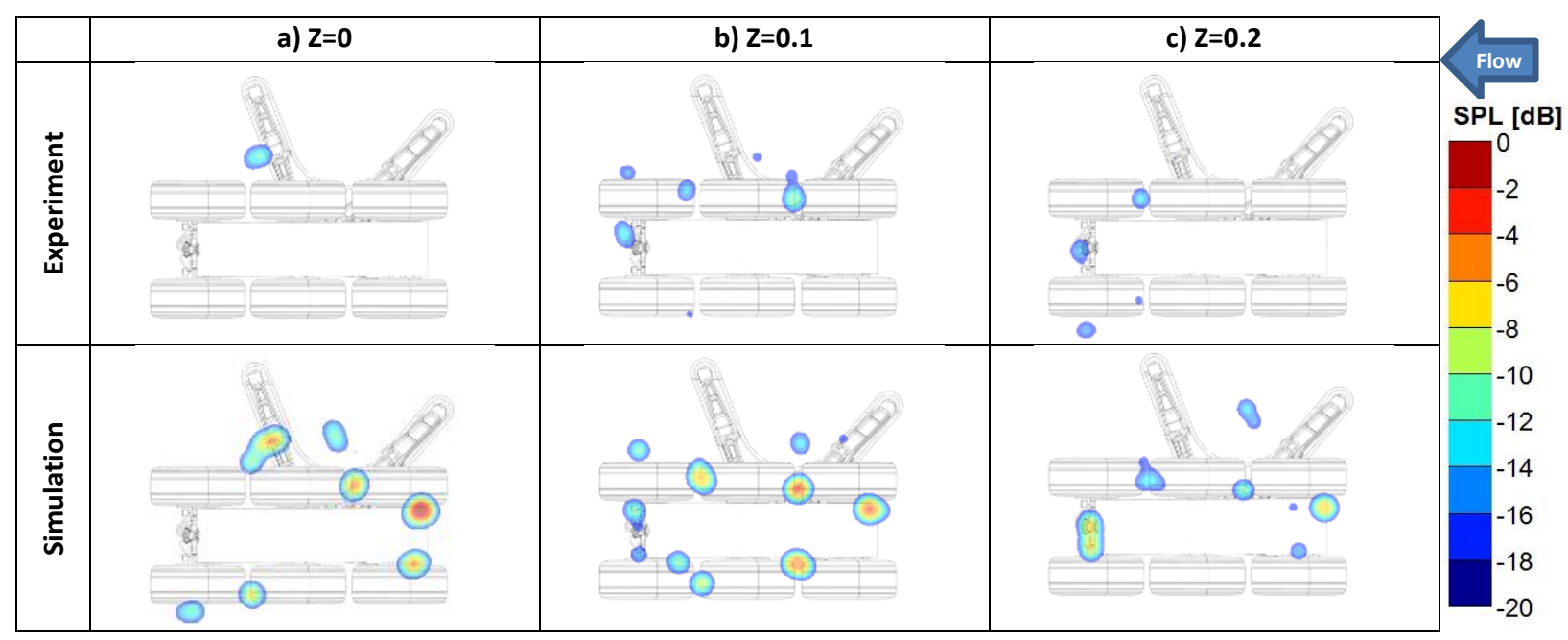

Fig. 20 Experimental and simulated (1/12th octave band) beamform maps with toboggan installed in overhead direction from CLEAN, $f=2.8 \mathrm{kHz}$.

While the beamform maps clearly highlight subtle differences between the measured and simulated sources, they fail to provide information on the underlying cause of the discrepancies. In our view, there are two plausible reasons for the observed discrepancy, both related to the differences in model setup between wind tunnel and free-air simulation. The first might be reflections from the wind tunnel walls. In its anechoic configuration, the VTSWT operates approximately like a closed-wall tunnel in terms of the flow field. This is especially true during acoustic measurements in the overhead direction, when only one wall is replaced with acoustically permeable Kevlar ${ }^{\circledR}$ fabric. The ceiling and floor are Kevlar-covered perforated plates. Although one wall remains acoustically hard, extensive experience testing in this facility indicates that reflections from the treated wind tunnel walls are practically nonexistent. This conjecture is difficult to test computationally given the complexities of the boundary conditions that would have to be developed for each wall.

A second, possible reason for the differences are changes in local flow acceleration and angularity caused by tunnel blockage effects that arise from the installed model. Airframe noise simulations for closed-wall facilities are avoided because strong sound reflections from the solid boundaries render the accurate prediction of farfield acoustic behavior virtually impossible. The present computational results were obtained from simulations of the gear model in free-air, thus neglecting blockage effects in the VTSWT. Because the $26 \%$-scale B777 main gear model is a complex geometry comprised of many bluff bodies of various shapes, sizes, and orientations, even relatively small differences in incoming flow angularity between the tested and simulated configurations may have a large impact on flow separation, vortex shedding, and wake-body interaction characteristics for the various gear components. To determine some of the flow field differences between closed-wall and free-air setups, the wind tunnel test section with the gear model installed was simulated. However, unlike the VTSWT in its anechoic configuration, all four test-section walls were treated as solid, inviscid boundaries (without acoustic treatment) in the computational setup. The simulation was conducted at a coarse-level resolution. For the purpose of aerodynamic comparison, which was the primary intent here, the selected resolution level was deemed sufficient [6] to highlight the flow differences/similarities between the tunnel and free-air setups.

Total velocity magnitude at a cross plane half-tire diameter forward of the front wheels is shown in Fig. 21. As anticipated, the local flow around the model in the wind tunnel simulation accelerates due to blockage effects. However, if the contours from the wind-tunnel simulation are normalized by the maximum velocity magnitude in the plane, a picture very similar to that of the free-air contours emerges (Fig. 22). Contours of lateral deflection-angle at the forward plane are shown in Figs. 23a and 23b. As expected, there are subtle differences between the two maps. The magnitude of the differences in lateral angles was quantified by subtracting wind-tunnel flow angles from the corresponding free-air values. The result, which has been plotted in Fig. 23c, indicates that the differences are within $\pm 1^{\circ}$. Vertical deflection angles are shown in Fig. 24. Again, differences in the contours between the two model setups are less than $\pm 1^{\circ}$.

Contours of velocity magnitude at a cross-plane cutting through the center of the middle wheels are presented in Fig. 25. As in the upstream plane, contours from the wind tunnel simulation show elevated speeds relative to free-air

American Institute of Aeronautics and Astronautics 
results in regions surrounding the model. To highlight the extent of the differences, the contours were scaled with the velocity magnitude extracted from the respective planes at the same locations relative to the gear model. The normalized contours, shown in Fig. 26, are remarkably similar and prove that the flow in both configurations produces comparable patterns. Normalized contours for a streamwise plane that bisects the vertical post and the longitudinal axle are presented in Fig. 27. These maps also show very similar patterns devoid of features that would suggest that the wind-tunnel setup produces major differences.

Simulated surface pressure fluctuations were band-filtered and the power spectral plots for the $2.748 \mathrm{kHz}$ to 2.911 kHz band are shown in Fig. 28 (bottom view) and Fig. 29 (side view), respectively. The surface pressure contours have been normalized by their respective dynamic pressures obtained from the same velocity magnitudes used in Fig. 26. While the contours in Figs. 28 and 29 look very similar, scrutiny of the surface pressures reveals that, even after normalization, the fluctuations for the wind-tunnel configuration have higher levels than those for free-air (Fig.30). The higher pressure levels observed for the wind tunnel simulation may have translated into higher farfield SPLs, which is contrary to the observed trend for the measured integrated spectrum in the $1.8 \mathrm{kHz}$ to $4 \mathrm{kHz}$ frequency range (Fig. 12a).

Since the comparison between free-air and wind-tunnel results was conducted for coarse-resolution simulations, an assessment of how the strength and distribution of the sources are affected by increments in spatial resolution would be very informative. A close-up view of the pressure contours on the landing gear axles and brakes from coarse-, medium-, and fine-resolution simulations of the free-air configuration [6] are shown in Fig.31. Notice that with increasing resolution, not only the pressure levels increase but also there are shifts in the distribution and relative strengths of prominent noise sources within each specific region. The underlying cause for these changes are the many sharp edges and smaller geometric features that get better resolved with improved spatial resolution. In the CAD representation of the model geometry, nearly all such edges and corners are well defined and represented as sharp. Depending on incoming local flow orientation, these sharp edges may produce high-amplitude pressure fluctuations. In the "as-built" gear model, however, such edges and corners were certainly manufactured with a fillet or chamfer that depended on the physical dimensions of the various sub-components being fabricated. The manner in which the edges are represented affects directly the overall accuracy, fidelity, and correspondence between simulated and tested model geometries. As an example, depending on the model manufacturing tolerances, many small gaps and openings may actually appear as either larger or closed-off when the physical model is assembled. Correspondingly, many of the extremely small gaps and openings may have been sealed off in the CAD geometry that was simulated to eliminate fictitious, high-frequency, sharp tones that result from severe under resolution.

Medium- and fine-resolution simulations are required to determine whether the redistribution and shift in the relative strength of the sources with increased resolution for the wind-tunnel configuration follows the same trend as that observed in the free-air setup. Unfortunately, these simulations are beyond the scope of the present effort.

As a final note, we observe that the fluctuating surface pressures shown in Figs. 28, 29, 30, and 31 are dominated by the hydrodynamic component of the pressure signal. There is no simple or clear way to decipher how and what portion of the hydrodynamic fluctuations gets converted to sound that is propagated to the farfield. Overall, the coarselevel results for off- and on-surface quantities examined here fail to reveal a compelling cause for the subtle discrepancy between measured and simulated spectra observed in the $1.8 \mathrm{kHz}$ to $4 \mathrm{kHz}$ frequency range. However, examination of spatial resolution effects on the fluctuating surface pressures for the free-air configuration suggests that small differences between simulated and tested model geometries are the likely cause of the discrepancy.

American Institute of Aeronautics and Astronautics 


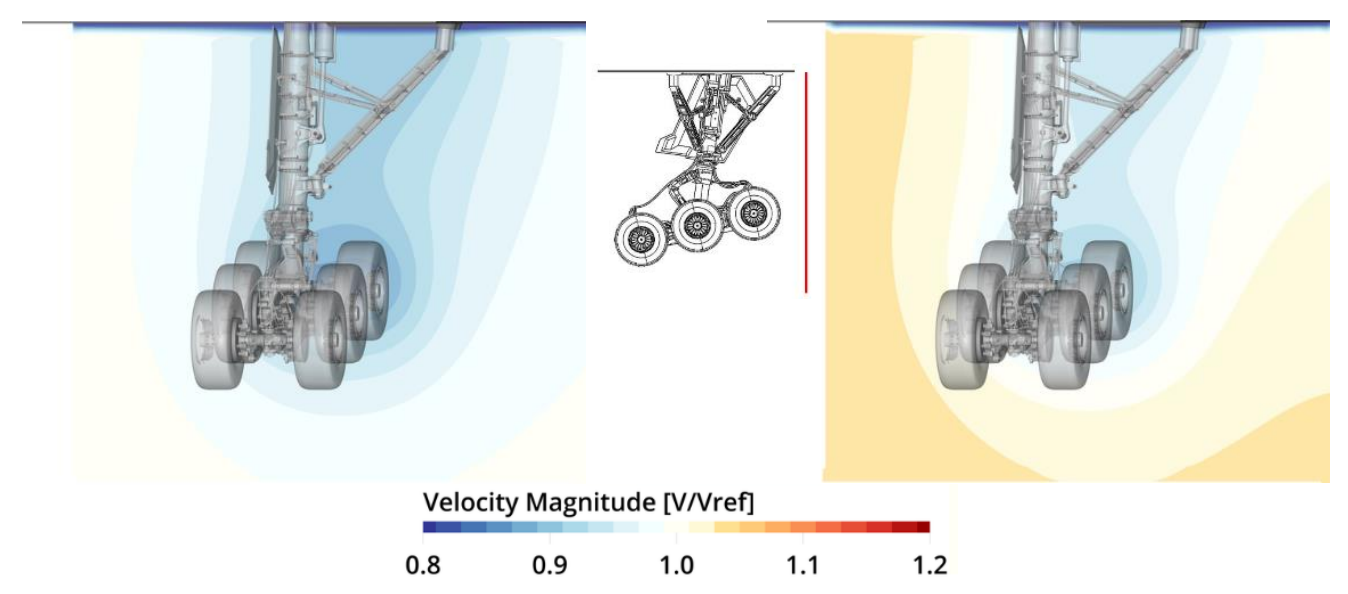

a) free-air simulation

b) wind-tunnel simulation

Fig. 21 Contours of velocity magnitude at a plane half a tire diameter forward of the front wheels.

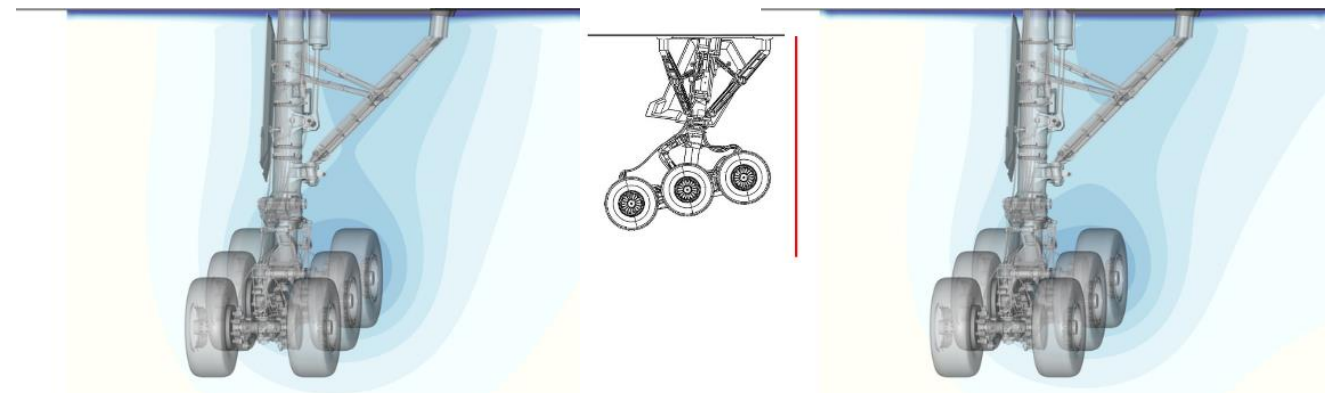

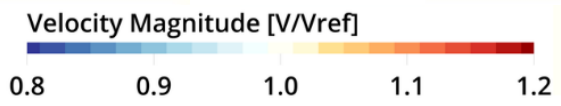
a) free-air simulation
b) wind-tunnel simulation

Fig. 22 Contours of normalized velocity magnitude at a plane half a tire diameter forward of the front wheels.
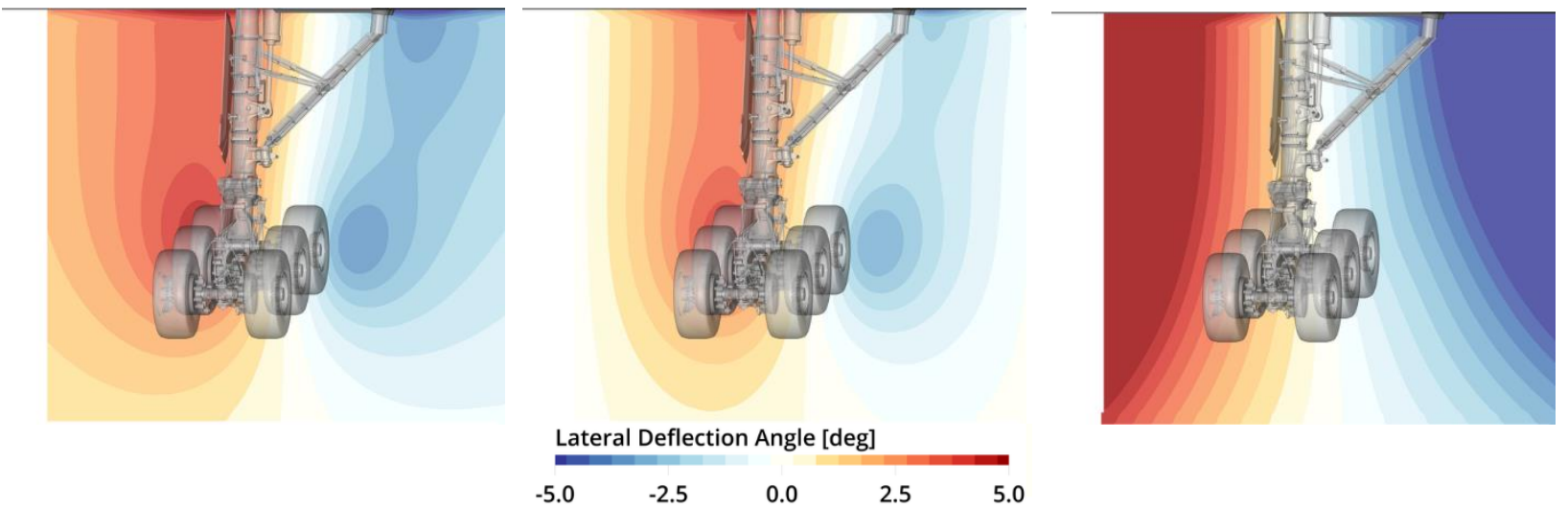

a) free-air simulation

b) wind-tunnel simulation

c) $\Delta$ free-air - wind tunnel

Fig. 23 Contours of lateral deflection angle beta at a plane half a tire diameter forward of the front wheels.

American Institute of Aeronautics and Astronautics 


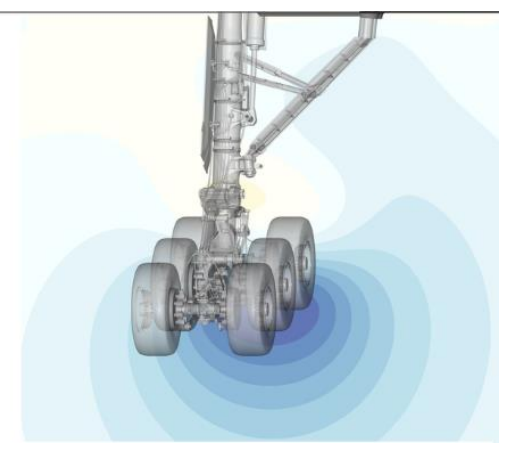

a) free-air simulation

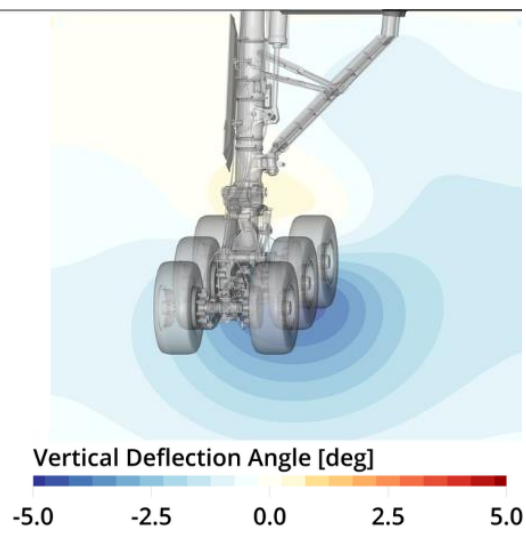

b) wind-tunnel simulation

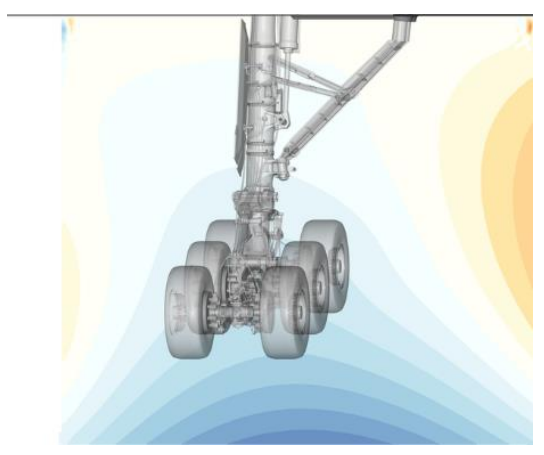

c) $\Delta$ free-air - wind tunnel

Fig. 24 Contours of vertical deflection angle alpha at a plane half a tire diameter forward of the front wheels.

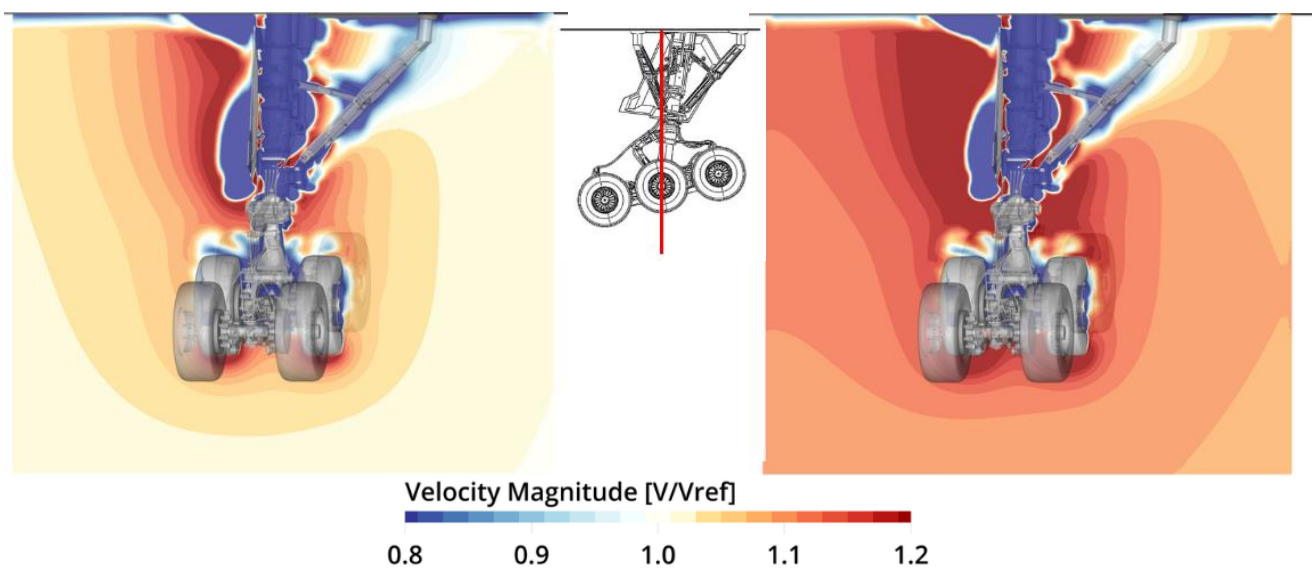

a) free-air simulation

b) wind-tunnel simulation

Fig. 25 Contours of velocity magnitude at a plane cutting through the center of the middle wheels.

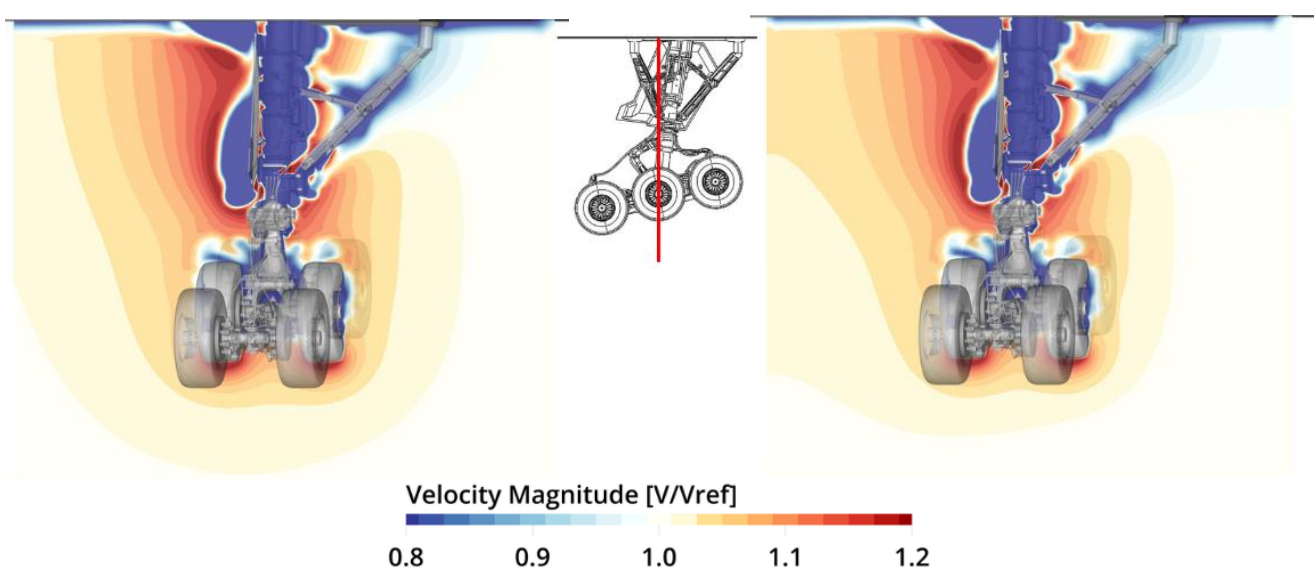

a) free-air simulation

b) wind-tunnel simulation

Fig. 26 Contours of normalized velocity magnitude at a plane cutting through the center of the middle wheels. 

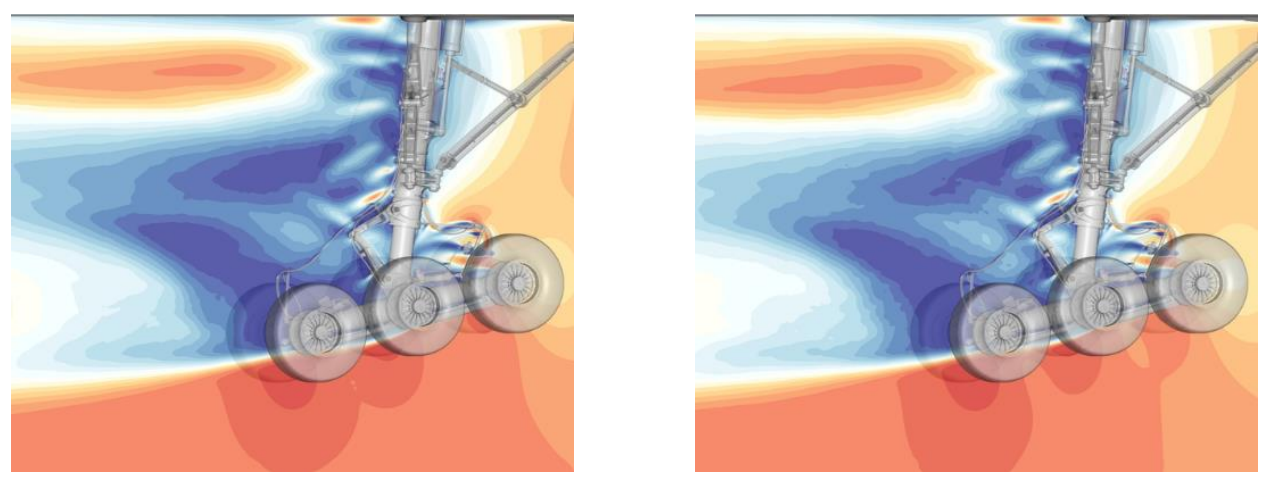

Velocity Magnitude [V/Vref]

$\begin{array}{lllll}0.2 & 0.4 & 0.7 & 0.9 & 1.2\end{array}$

a) free-air simulation

b) wind-tunnel simulation

Fig. 27 Contours of normalized velocity magnitude at a plane bisecting vertical post and longitudinal axle.

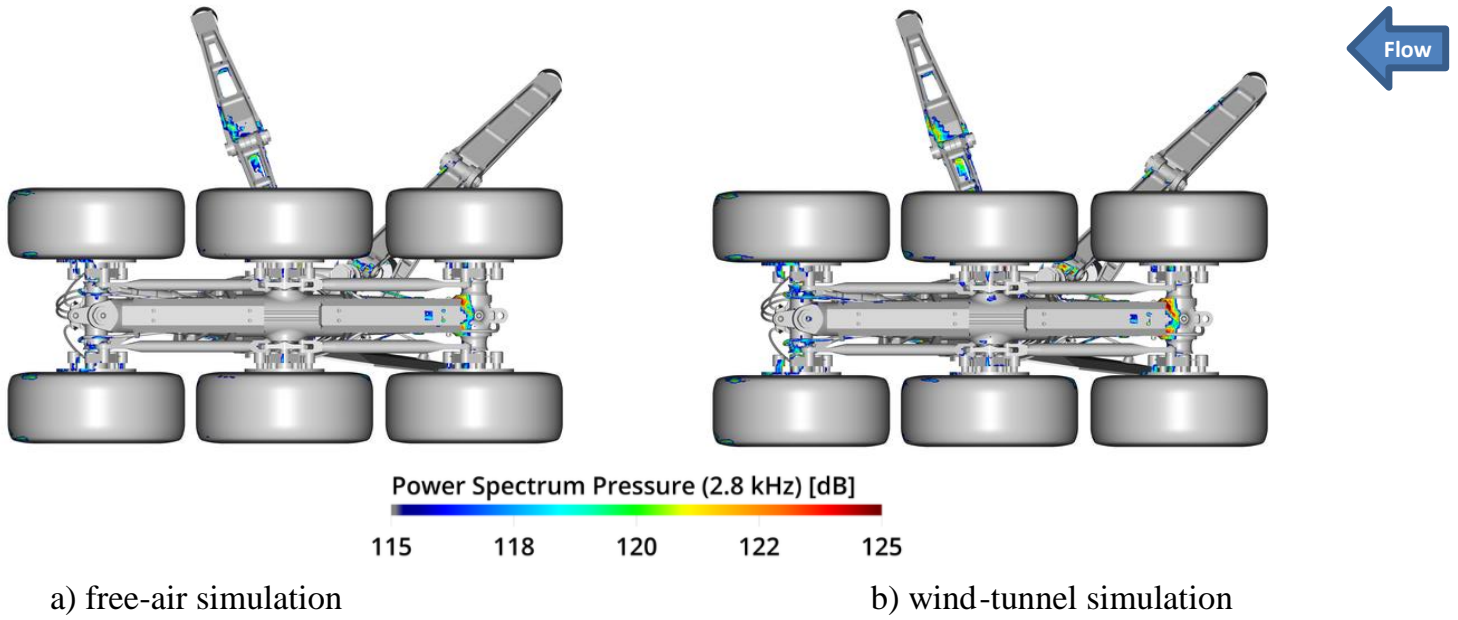

Fig. 28 Bottom view of band-filtered power spectral levels for normalized surface pressure fluctuations.

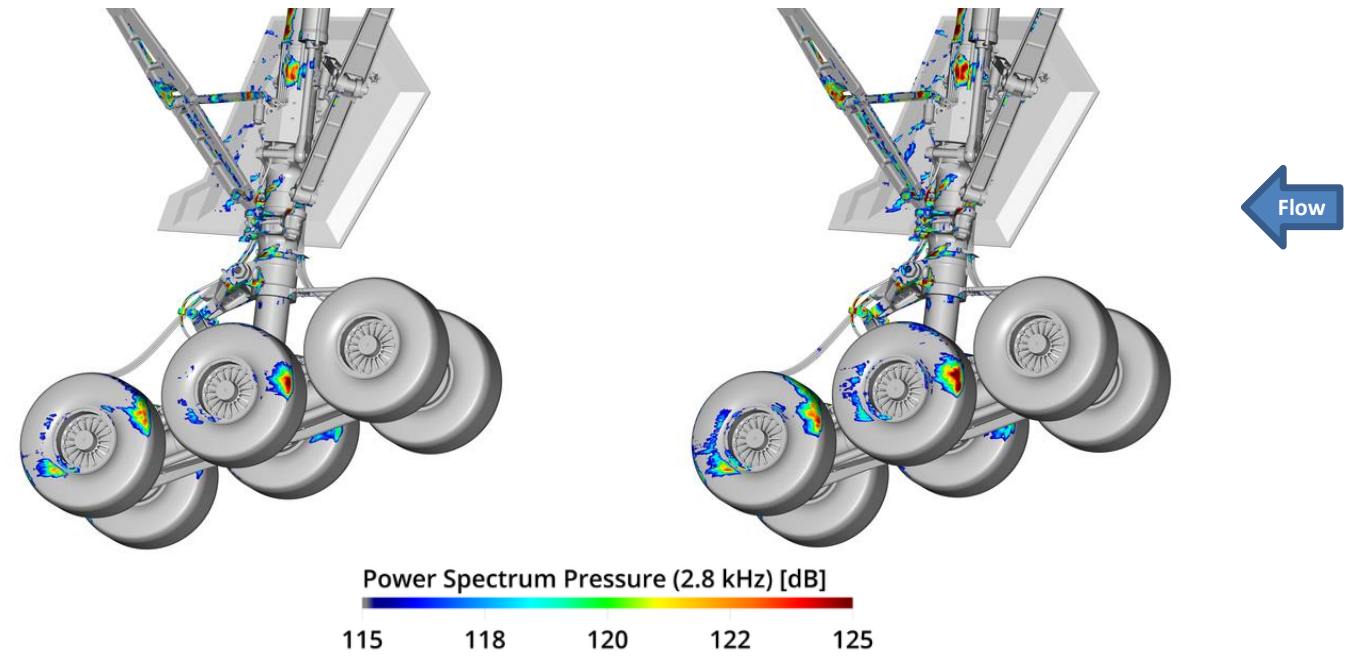
a) free-air simulation
b) wind-tunnel simulation

Fig. 29 Side view of band-filtered power spectral levels for normalized surface pressure fluctuations.

American Institute of Aeronautics and Astronautics 


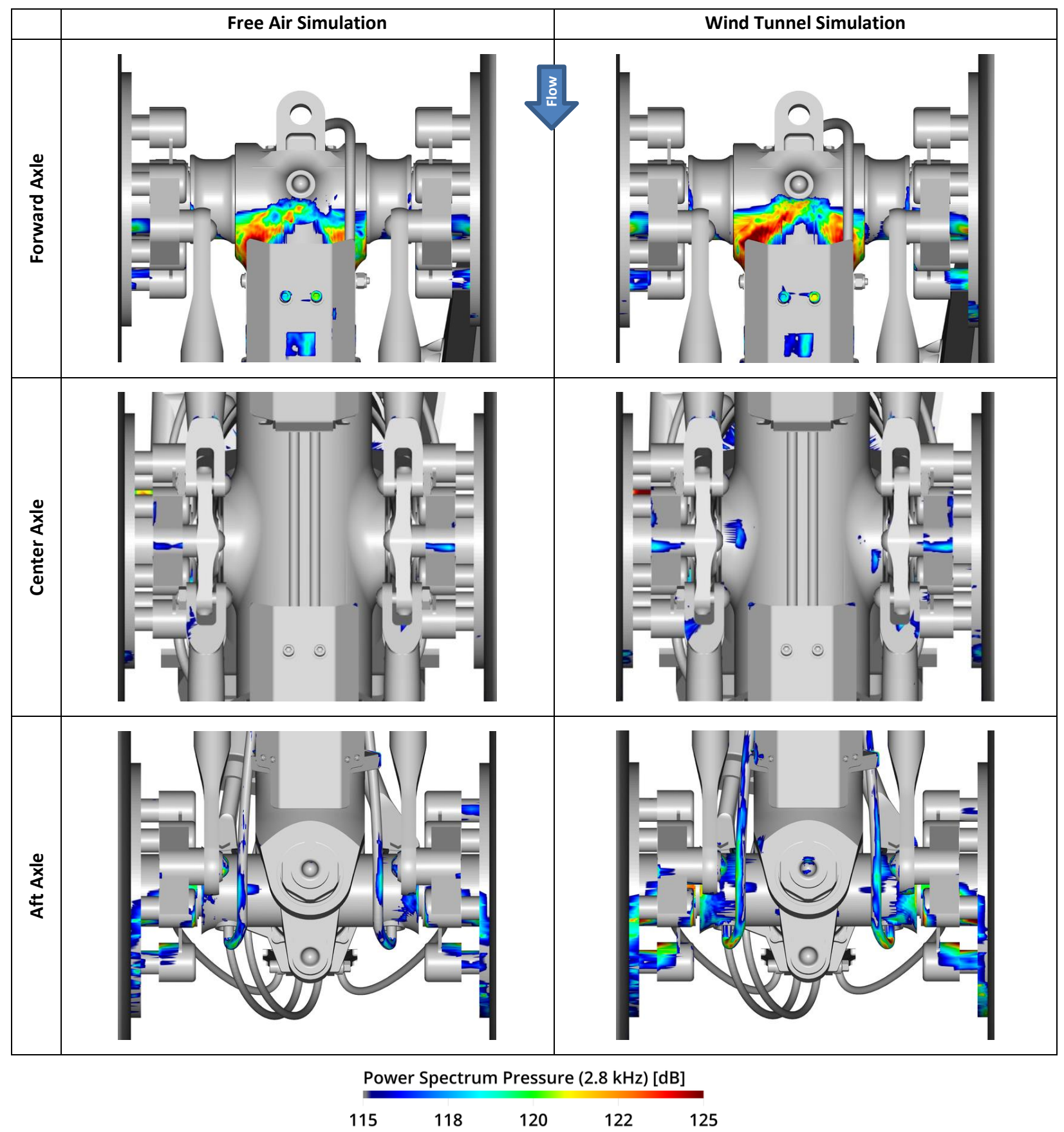

Fig. 30 Close-up view of band-filtered power spectral levels for normalized surface pressure fluctuations in the vicinity of the landing gear axles. Flow direction is top to bottom. 


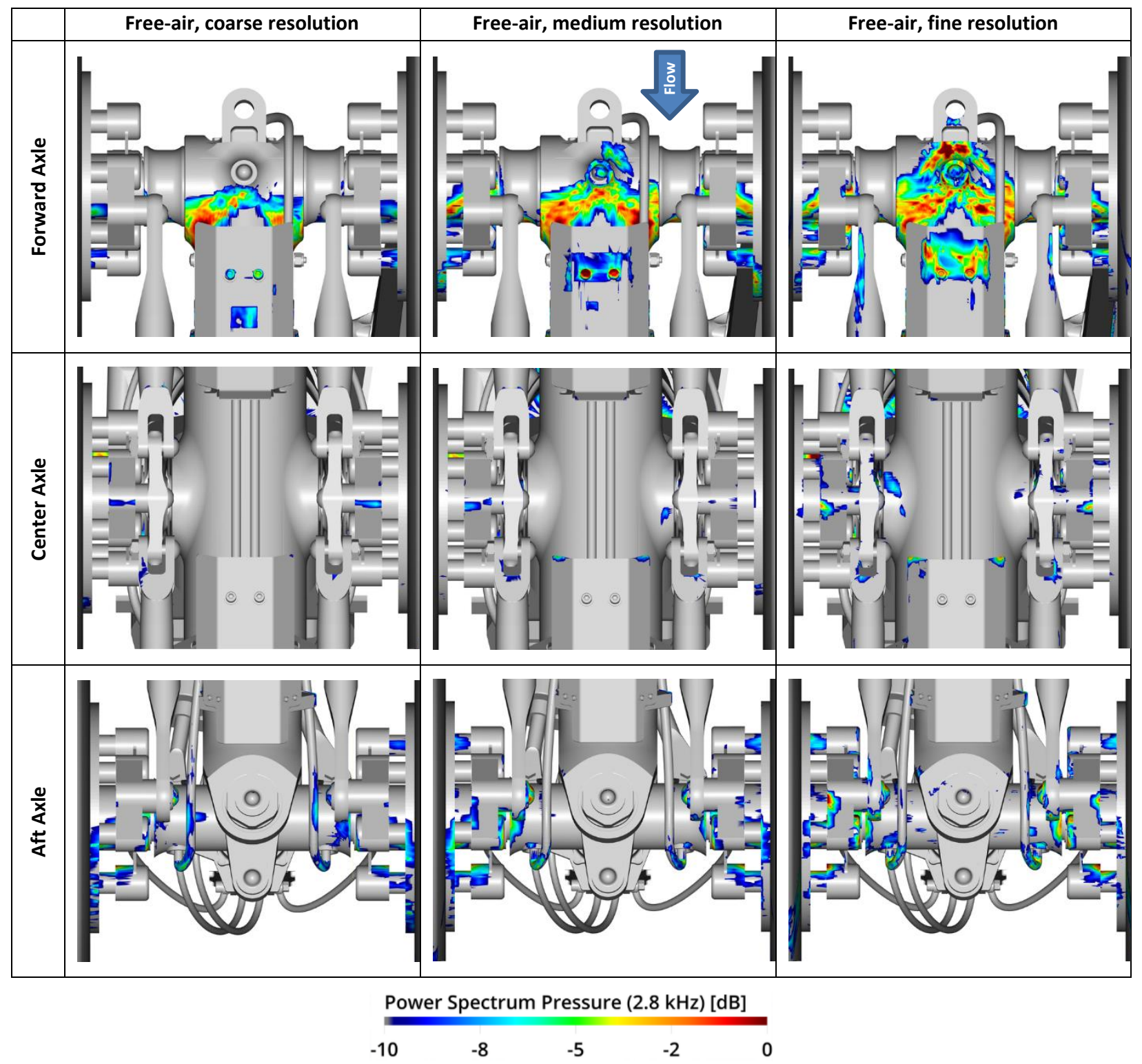

Fig. 31 Close-up view of band-filtered power spectral levels for normalized surface pressure fluctuations in the vicinity of the landing gear axles. Flow direction is top to bottom.

\section{Conclusions}

An extensive analysis of previously measured and simulated phased array data for the NASA $26 \%$-scale B777200 aircraft main landing gear model was performed for gear configurations with and without a toboggan noise reduction fairing. Acoustic data reprocessing was initiated to address the deficiencies encountered in the source localization (beamform) maps and the integrated farfield spectra at mid to high frequencies in our previous study, where conventional array processing techniques were applied to the same data sets. To eliminate the major shortcomings of the previous analysis, the CLEAN processing technique in 3D was applied to the acoustic data sets. Application of the CLEAN approach resulted in higher quality beamform maps with many of the previously observed side lobes either eliminated or significantly suppressed. As a result, integration of the experimentally and computationally based maps showed substantial improvement in the quality of the high-frequency spectral content relative to the corresponding spectra generated via conventional array processing, thus extending the good agreement achieved between measured and predicted farfield spectra from $8 \mathrm{kHz}$ to $14 \mathrm{kHz}$ in model-scale frequencies.

American Institute of Aeronautics and Astronautics 
Despite the good agreement achieved, measured and predicted farfield spectra show subtle discrepancies in the $1.8 \mathrm{kHz}$ to $4.0 \mathrm{kHz}$ model-scale frequency range. The differences were conjectured to be caused by model blockage effects in the wind-tunnel setup that were not present in the free-air setup used for simulation. To determine the causes of some flow field differences between the closed-wall and free-air setups, a hard-wall wind tunnel test section with the gear model installed was simulated. An in-depth comparison of aerodynamic results for free-air and wind tunnel configurations for the off-surface flow field (velocity contours and flow angularity) and band-filtered surface pressure fluctuations was attempted. While the hard-wall tunnel simulation produced higher-velocity local flows in the vicinity of the model caused by blockage effects, the overall flow patterns and features remained remarkably similar to those obtained from the free-air simulation. Based on close examination of the fluctuating surface pressures on the gear truck, the discrepancies observed in the farfield spectra for the $1.8 \mathrm{kHz}$ to $4.0 \mathrm{kHz}$ frequency range are conjectured to be attributed to small differences between simulated and tested model geometries.

\section{Acknowledgments}

This work was supported by the Flight Demonstrations and Capabilities (FDC) project under the Integrated Aviation Systems Program (IASP) of the NASA Aeronautics Research Mission Directorate. All simulations were performed in the Pleiades supercomputer at the NASA Advanced Supercomputing (NAS) facility at Ames Research Center. The logistical support provided by NAS staff is greatly appreciated.

\section{References}

[1] Dobrzynski, W., “Almost 40 Years of Airframe Noise Research: What Did We Achieve,” J. Aircraft, Vol. 47, No. 2, MarchApril 2010, pp. 353-367.

[2] Khorrami, M. R., Fares, E., and Casalino, D., "Towards Full-Aircraft Airframe Noise Prediction: Lattice-Boltzmann Simulations," AIAA Paper 2014-2481, June 2014.

[3] Khorrami, M. R., and Fares, E., "Simulation-Based Airframe Noise Prediction of a Full-Scale Full Aircraft," AIAA Paper 20162706, May-June 2016.

[4] Fares, E., Duda, B., and Khorrami, M. R., “Airframe Noise Prediction of a Detailed Full Aircraft in Model and Full Scale Using a Lattice Boltzmann Approach,” AIAA Paper 2016-2707, May-June 2016.

[5] Ravetta, P. A., Khorrami, M. R., Burdisso, R. A., and Wisda, D. M., “Acoustic Measurements of a Large Civil Transport Main Landing Gear Model," AIAA Paper 2016-2901, May-June 2016.

[6] Konig, B., Fares, E. Ravetta, P. A., and Khorrami, M. R., “A Comparative Study of Simulated and Measured Main Landing Gear Noise for Large Civil Transports,” AIAA Paper 2017-3013, June 2017.

[7] Jaeger, S. M., Burnside, N. J., Soderman, P. T., Horne, W. C., and James, K. D., "Microphone Array Assessment of an Isolated, 26\%-Scale, High Fidelity Landing Gear," AIAA Paper 2002-2410, June 2002.

[8] Ravetta, P. A., Burdisso, R. A., and Ng, W. F., "Wind Tunnel Aeroacoustic Measurements of a 26\%-scale 777 Main Landing Gear Model,” AIAA Paper 2004-2885, May 2004.

[9] Ravetta, P. A., Burdisso, R. A., Ng, W. F., Khorrami, M. R., and Stoker, R. W., "Screening of Potential Noise Control Devices at Virginia Tech for QTD II Flight Test," AIAA Paper 2007-3455, May 2007.

[10] Remillieux, M. C., Camargo, H. E., Ravetta, P. A., Burdisso, R. A., and Ng, W. F., "Novel Kevlar-Walled Wind Tunnel for Aeroacoustic Testing of a Landing Gear," AIAA Journal, Vol. 46, No. 7, July 2008, pp. 1631-1639.

[11] Horne, W. C., James, K. D., Arledge, T. K., Soderman, P. T., Burnside, N., and Jaeger, S. M., "Measurements of 26\%-scale 777 Airframe Noise in the NASA Ames 40- by 80-Foot Wind Tunnel," AIAA Paper 2005-2810, May 2005.

[12] Hawking, J. E., and Ffowcs Williams, D. L., "Sound Generation by Turbulence and Surfaces in Arbitrary Motion," Philosophical Transactions of the Royal Society of London, Series A, Mathematical and Physical Sciences, Vol. 264, No. 1151, 1969, pp. 321-342.

[13] Sijtsma, P., "CLEAN based on Spatial Source Coherence," AIAA Paper 2007-3436, May 2007.

[14] Devenport, W. J., Burdisso, R. A., Borgoltz, A., Ravetta P. A., Barone, M. F., Brown, K. A., and Morton, M. A., "The Kevlarwalled anechoic wind tunnel, "Journal of Sound and Vibration, Vol. 332, No. 17, August 2013, pp. 3971-3991.

[15] Mueller, T. (ed.), Aeroacoustic Measurements, Springer, 2002. ISBN 3-540-41757-5.

American Institute of Aeronautics and Astronautics 